\title{
GLOBAL WELLPOSEDNESS AND LARGE TIME BEHAVIOR OF SOLUTIONS TO THE $N$-DIMENSIONAL COMPRESSIBLE OLDROYD-B MODEL
}

\author{
XIAOPING ZHAI ${ }^{\dagger}$ AND YONGSHENG LI
}

\begin{abstract}
The purpose of this work is to study the global wellposedness and large time behavior results of strong solutions for the compressible Oldroyd-B model derived by Barrett, Lu, Süli (Commun. Math. Sci., 15, 1265-1323, 2017). Exploiting the Harmonic analysis tools (especially Littlewood-Paley theory), we first study the global well-posedness of the model with small initial data in spaces with low regularity. Then, under a suitable condition involving only the low frequency of the initial data, we also obtain the optimal decay rates of the solutions. Compared with the result by Wang and Wen (Math. Models Methods Appl. Sci., 30, 139$179,2020)$, the polymer number density is allowed to vanish and the stress tensor isnear zero equilibrium.
\end{abstract}

\section{INTRODUCTION AND THE MAIN RESULTS}

In this paper, we mainly consider the Cauchy problem of the following compressible OldroydB model:

$$
\left\{\begin{array}{l}
\partial_{t} \rho+\operatorname{div}(\rho \mathbf{u})=0, \\
\partial_{t} \eta+\operatorname{div}(\eta \mathbf{u})-\varepsilon \Delta \eta=0, \quad x \in \mathbb{R}^{n}, \quad t>0, \\
\partial_{t} \mathbb{T}+(\mathbf{u} \cdot \nabla) \mathbb{T}+\mathbb{T} \operatorname{div} \mathbf{u}-\left(\nabla \mathbf{u} \mathbb{T}+\mathbb{T} \nabla^{\top} \mathbf{u}\right)-\varepsilon \Delta \mathbb{T}=\frac{\kappa A_{0}}{2 \lambda_{1}} \eta \mathbb{I}-\frac{A_{0}}{2 \lambda_{1}} \mathbb{T}, \\
\rho\left(\partial_{t} \mathbf{u}+\mathbf{u} \cdot \nabla \mathbf{u}\right)-\mu \Delta \mathbf{u}-(\lambda+\mu) \nabla \operatorname{div} \mathbf{u}+\nabla P=\operatorname{div}\left(\mathbb{T}-\left(\kappa L \eta+\zeta \eta^{2}\right) \mathbb{I}\right),
\end{array}\right.
$$

for $(t, x) \in \mathbb{R}_{+} \times \mathbb{R}^{n}(n=2,3)$. Here $\rho=\rho(t, x) \in \mathbb{R}_{+}$is the density function of the fluid, $\mathbf{u}=\mathbf{u}(t, x) \in \mathbb{R}^{n}$ is the velocity. The symmetric matrix function $\mathbb{T}=\left(\mathbb{T}_{i, j}\right), 1 \leq i, j \leq n$ is the extra stress tensor and $\eta=\eta(t, x) \in \mathbb{R}_{+}$represents the polymer number density defined as the integral of a probability density function $\psi$ with respect to the conformation vector, which is a microscopic variable in the modeling of dilute polymer chains, i.e.,

$$
\eta=\int_{\mathbb{R}^{n}} \psi(t, x, q) d q \geq 0
$$

Here $\psi$ is governed by the Fokker-Plank equation.

The viscosities constant $\mu$ and $\lambda$ are supposed to satisfy $\mu>0$ and $n \lambda+2 \mu \geq 0$. In particular, the parameters $\kappa, \varepsilon, A_{0}, \lambda_{1}$ are all positive numbers, whereas $\zeta \geq 0$ and $L \geq 0$ with $\zeta+L \neq 0$. The term $\kappa L \eta+\zeta \eta^{2}$ in the momentum equation (1.1) can be seen as the polymer pressure, compared to the fluid pressure $P(\rho)=A \rho^{\gamma}$. System (1.1) is supplemented with the initial data

$$
\left.(\rho, \mathbf{u}, \eta, \mathbb{T})\right|_{t=0}=\left(\rho_{0}(x), \mathbf{u}_{0}(x), \eta_{0}(x), \mathbb{T}_{0}(x)\right), x \in \mathbb{R}^{n},
$$

and with far field behaviors

$$
\rho \rightarrow \bar{\rho}, \quad \mathbf{u} \rightarrow \mathbf{0}, \quad \eta \rightarrow 0, \quad \mathbb{T} \rightarrow 0 \mathbb{I} \quad \text { as } \quad|x| \rightarrow \infty .
$$

Micro-macro models of dilute polymeric fluids that arise from statistical physics are based on coupling the Navier- Stokes system to the Fokker-Planck equation. In these models polymer molecules are idealized as chains of massless beads, linearly connected with inextensible rods 
or elastic springs. The model we consider here was first derived by Barrett, Lu, and Süli [3] via micro-macro analysis of the compressible Navier-Stokes-Fokker-Planck system studied in a series of papers by Barrett and Süli [2]-7. Barrett and Süli [3] obtained the global-in-time finite-energy weak solutions with large initial data in $\mathbb{R}^{2}$. However, the uniqueness of the global weak solution is still open. Later, Lu and Zhang 34 proved the local wellposedness, weakstrong uniqueness and a refined blow-up criterion involving only the upper bound of the fluid density. Wang and Wen [43] obtained the global wellposedness of (1.1) as well as associated time-decay estimates in Sobolev space, if the initial data is near a nonzero equillbrium state. Most recently, the first author of the present paper in [45] justify the low Mach number convergence to the incompressible Oldoryd-B model for viscous compressible Oldoryd-B model in the ill-prepared data case. When neglecting the stress diffusion in (1.1) and assuming further the extra stress tensor is a scalar matrix, Lu and Pokorný [33] obtained the global weak solutions with large data.

It is interesting to note that the model (1.1) with $\eta=0$ is related to the following compressible Oldoryd-B model, i.e.,

$$
\left\{\begin{array}{l}
\partial_{t} \rho+\operatorname{div}(\rho \mathbf{u})=0 \\
\rho\left(\partial_{t} \mathbf{u}+(\mathbf{u} \cdot \nabla) \mathbf{u}\right)-\mu \Delta \mathbf{u}-(\lambda+\mu) \nabla \operatorname{div} \mathbf{u}+\nabla P=\mu_{1} \operatorname{div} \mathbb{T} \\
\partial_{t} \mathbb{T}+(\mathbf{u} \cdot \nabla) \mathbb{T}+g(\mathbb{T}, \nabla \mathbf{u})+\beta \mathbb{T}=\mu_{2} D(\mathbf{u}), \quad x \in \mathbb{R}^{n}, \quad t>0
\end{array}\right.
$$

where $D(\mathbf{u})$ is the symmetric part of $\nabla \mathbf{u}$, and $\Omega(\mathbf{u})$ is the skew-symmetric part of $\nabla \mathbf{u}$, namely

$$
D(\mathbf{u})=\frac{1}{2}\left(\nabla \mathbf{u}+(\nabla \mathbf{u})^{\top}\right), \quad \Omega(\mathbf{u})=\frac{1}{2}\left(\nabla \mathbf{u}-(\nabla \mathbf{u})^{\top}\right)
$$

and

$$
g(\mathbb{T}, \nabla \mathbf{u}) \stackrel{\text { def }}{=} \mathbb{T} \Omega(\mathbf{u})-\Omega(\mathbf{u}) \mathbb{T}-b(D(\mathbf{u}) \mathbb{T}+\mathbb{T} D(\mathbf{u})), \quad b \text { is a parameter in }[-1,1] .
$$

The Oldroyd-B model attracts continuous attentions of mathematicians, however, there has few known results concerning compressible Oldroyd-B models of (1.4). Lei [27] and Gullopé et al. 20] studied the incompressible limit problem of the compressible Oldroyd-B model in a torus and bounded domain of $\mathbb{R}^{3}$, respectively. Recently, Zi [49] obtained the global small solutions of (1.4) in the critical $L^{2}$ Besov spaces. The first author in the present paper and Chen [46] generalized the result of [49] about (1.4) without damping mechanism to the critical $L^{p}$ spaces. For the compressible Oldroyd type model based on the deformation tensor, see the results [25], [26], 37], 39] and references therein. Let $\rho$ be constant, the system (1.4) reduces to be the incompressible Oldroyd-B model, which has made rather rich results, see [11], [19], [21], [22], [30], [40].

Let us give now more details on the form of the solutions that we are going to consider. Let $\bar{\rho}=1$ in (1.3) and define $\rho=1+a$, we can reformulate the system (1.1) into the following form:

$$
\left\{\begin{array}{l}
\partial_{t} a+\operatorname{div} \mathbf{u}=-\operatorname{div}(a \mathbf{u}) \\
\partial_{t} \eta-\varepsilon \Delta \eta=-\operatorname{div}(\eta \mathbf{u}) \\
\partial_{t} \mathbb{T}+\frac{A_{0}}{2 \lambda_{1}} \mathbb{T}+(\mathbf{u} \cdot \nabla) \mathbb{T}-\varepsilon \Delta \mathbb{T}=\frac{\kappa A_{0}}{2 \lambda_{1}} \eta \mathbb{I}+F(\mathbb{T}, \mathbf{u}), \\
\partial_{t} \mathbf{u}+\mathbf{u} \cdot \nabla \mathbf{u}-\mu \Delta \mathbf{u}-(\lambda+\mu) \nabla \operatorname{div} \mathbf{u}+\nabla a=\operatorname{div} \mathbb{T}-\kappa L \nabla \eta+G(a, \mathbf{u}, \eta, \mathbb{T}), \\
\left.(a, \mathbf{u}, \eta, \mathbb{T})\right|_{t=0}=\left(a_{0}(x), \mathbf{u}_{0}(x), \eta_{0}(x), \mathbb{T}_{0}(x)\right),
\end{array}\right.
$$

with

$$
\nu \stackrel{\text { def }}{=} \lambda+2 \mu, \quad I(a) \stackrel{\text { def }}{=} \frac{a}{1+a}, \quad k(a) \stackrel{\text { def }}{=}-\frac{P^{\prime}(1+a)}{1+a}+P^{\prime}(1) \quad \text { with } P^{\prime}(1)=1,
$$




$$
\begin{aligned}
F(\mathbb{T}, \mathbf{u}) & \stackrel{\text { def }}{=}\left(\nabla \mathbf{u} \mathbb{T}+\mathbb{T} \nabla^{\top} \mathbf{u}\right)-\mathbb{T} \operatorname{div} \mathbf{u}, \\
G(a, \mathbf{u}, \eta, \mathbb{T}) & \stackrel{\text { def }}{=} k(a) \nabla a-I(a)(\mu \Delta \mathbf{u}+(\lambda+\mu) \nabla \operatorname{div} \mathbf{u})-I(a)(\operatorname{div} \mathbb{T}-\nabla \eta)-\zeta(1-I(a)) \eta \nabla \eta .
\end{aligned}
$$

The first main result of the paper is stated as follows.

Theorem 1.1. (Local wellposedness) Let $n=2,3$ and $1<p<2 n$. For any $\mathbf{u}_{0} \in \dot{B}_{p, 1}^{\frac{n}{p}-1}\left(\mathbb{R}^{n}\right)$, $\left(\eta_{0}, \mathbb{T}_{0}\right) \in \dot{B}_{p, 1}^{\frac{n}{p}}\left(\mathbb{R}^{n}\right)$ and $a_{0} \in \dot{B}_{p, 1}^{\frac{n}{p}}\left(\mathbb{R}^{n}\right)$ with $1+a_{0}$ bounded away from zero. Then there exists a positive time $T$ such that the system (1.5) has a unique solution with

$$
\begin{aligned}
& a \in C_{b}\left([0, T] ; \dot{B}_{p, 1}^{\frac{n}{p}}\right), \quad \mathbf{u} \in C_{b}\left([0, T] ; \dot{B}_{p, 1}^{\frac{n}{p}-1}\right) \cap L^{1}\left([0, T] ; \dot{B}_{p, 1}^{\frac{n}{p}+1}\right), \\
& (\eta, \mathbb{T}) \in C_{b}\left([0, T] ; \dot{B}_{p, 1}^{\frac{n}{p}}\right) \cap L^{1}\left([0, T] ; \dot{B}_{p, 1}^{\frac{n}{p}+2}\right), \quad \mathbb{T} \in L^{1}\left([0, T] ; \dot{B}_{p, 1}^{\frac{n}{p}}\right) .
\end{aligned}
$$

Remark 1.2. The solutions constructed here allow the regularity exponent $n / p-1$ for the velocity becomes negative. Our result thus applies to large highly oscillating initial velocities (see [12] for more explanation).

Before presenting the second result of the paper, we give the following notation. Let $\mathcal{S}\left(\mathbb{R}^{n}\right)$ be the space of rapidly decreasing functions over $\mathbb{R}^{n}$ and $\mathcal{S}^{\prime}\left(\mathbb{R}^{n}\right)$ its dual space. For any $z \in \mathcal{S}^{\prime}\left(\mathbb{R}^{n}\right)$, the low and high frequency parts are expressed as

$$
z^{\ell} \stackrel{\text { def }}{=} \sum_{j \leq j_{0}} \dot{\Delta}_{j} z \quad \text { and } \quad z^{h} \stackrel{\text { def }}{=} \sum_{j>j_{0}} \dot{\Delta}_{j} z
$$

for some fixed integer $j_{0} \geq 1$ (the value of which follows from the proof of the main theorems). The corresponding truncated semi-norms are defined as follows:

$$
\|z\|_{\dot{B}_{p, r}^{s}}^{\ell} \stackrel{\text { def }}{=}\left\|z^{\ell}\right\|_{\dot{B}_{p, r}^{s}} \text { and }\|z\|_{\dot{B}_{p, r}^{s}}^{h} \stackrel{\text { def }}{=}\left\|z^{h}\right\|_{\dot{B}_{p, r}^{s}} .
$$

Denote

$$
\Lambda \stackrel{\text { def }}{=} \sqrt{-\Delta}, \quad \text { and } \quad \mathcal{P}=\mathcal{I}-\mathcal{Q} \stackrel{\text { def }}{=} \mathcal{I}-\nabla \Delta^{-1} \operatorname{div} .
$$

The second main result of the paper is stated as follows.

Theorem 1.3. (Global wellposedness) Let $n=2,3$ and

$$
2 \leq p \leq \min (4,2 n /(n-2)) \quad \text { and, additionally, } p \neq 4 \text { if } n=2 .
$$

For any $\left(a_{0}^{\ell}, \mathbf{u}_{0}^{\ell}\right) \in \dot{B}_{2,1}^{\frac{n}{2}-1}\left(\mathbb{R}^{n}\right), \eta_{0}^{\ell} \in \dot{B}_{2,1}^{\frac{n}{2}-2}\left(\mathbb{R}^{n}\right), \mathbb{T}_{0}^{\ell} \in \dot{B}_{2,1}^{\frac{n}{2}}\left(\mathbb{R}^{n}\right)$, and $\left(a_{0}^{h}, \mathbb{T}_{0}^{h}\right) \in \dot{B}_{p, 1}^{\frac{n}{p}}\left(\mathbb{R}^{n}\right)$, $\left(\mathbf{u}_{0}^{h}, \eta_{0}^{h}\right) \in \dot{B}_{p, 1}^{\frac{n}{p}-1}\left(\mathbb{R}^{n}\right)$. There exists a positive constant $c_{0}$ such that if,

$$
\left\|\left(a_{0}^{\ell}, \mathbf{u}_{0}^{\ell}\right)\right\|_{\dot{B}_{2,1}^{\frac{n}{2}-1}}+\left\|\mathbb{T}_{0}^{\ell}\right\|_{\dot{B}_{2,1}^{\frac{n}{2}}}+\left\|\eta_{0}^{\ell}\right\|_{\dot{B}_{2,1}^{\frac{n}{2}-2}}+\left\|\left(a_{0}^{h}, \mathbb{T}_{0}^{h}\right)\right\|_{\dot{B}_{p, 1}^{\frac{n}{p}}}+\left\|\left(\mathbf{u}_{0}^{h}, \eta_{0}^{h}\right)\right\|_{\dot{B}_{p, 1}^{\frac{n}{p}-1}} \leq c_{0},
$$

then the system (1.5) has a unique global solution $(a, u, \eta, \mathbb{T})$ so that

$$
\begin{array}{ll}
a^{\ell} \in C_{b}\left(\mathbb{R}^{+} ; \dot{B}_{2,1}^{\frac{n}{2}-1}\right) \cap L^{1}\left(\mathbb{R}^{+} ; \dot{B}_{2,1}^{\frac{n}{2}+1}\right), & a^{h} \in C_{b}\left(\mathbb{R}^{+} ; \dot{B}_{p, 1}^{\frac{n}{p}}\right) \cap L^{1}\left(\mathbb{R}^{+} ; \dot{B}_{p, 1}^{\frac{n}{p}}\right), \\
\mathbf{u}^{\ell} \in C_{b}\left(\mathbb{R}^{+} ; \dot{B}_{2,1}^{\frac{n}{2}-1} \cap L^{1}\left(\mathbb{R}^{+} ; \dot{B}_{2,1}^{\frac{n}{2}+1}\right),\right. & \mathbf{u}^{h} \in C_{b}\left(\mathbb{R}^{+} ; \dot{B}_{p, 1}^{\frac{n}{p}-1} \cap L^{1}\left(\mathbb{R}^{+} ; \dot{B}_{p, 1}^{\frac{n}{p}+1}\right),\right. \\
\eta^{\ell} \in C_{b}\left(\mathbb{R}^{+} ; \dot{B}_{2,1}^{\frac{n}{2}-2}\right) \cap L^{1}\left(\mathbb{R}^{+} ; \dot{B}_{2,1}^{\frac{n}{2}}\right), & \eta^{h} \in C_{b}\left(\mathbb{R}^{+} ; \dot{B}_{p, 1}^{\frac{n}{p}-1}\right) \cap L^{1}\left(\mathbb{R}^{+} ; \dot{B}_{p, 1}^{\frac{n}{p}+1}\right), \\
\mathbb{T}^{\ell} \in C_{b}\left(\mathbb{R}^{+} ; \dot{B}_{2,1}^{\frac{n}{2}}\right) \cap L^{1}\left(\mathbb{R}^{+} ; \dot{B}_{2,1}^{\frac{n}{2}}\right), & \mathbb{T}^{h} \in C_{b}\left(\mathbb{R}^{+} ; \dot{B}_{p, 1}^{\frac{n}{p}}\right) \cap L^{1}\left(\mathbb{R}^{+} ; \dot{B}_{p, 1}^{\frac{n}{p}+2}\right) .
\end{array}
$$

Moreover, there exists some constant $C$ such that

$$
X(t) \leq C c_{0}
$$


with

$$
\begin{gathered}
X(t) \stackrel{\text { def }}{=}\|(a, \mathbf{u})\|_{\widetilde{L}_{t}^{\infty}\left(\dot{B}_{2,1}^{\frac{n}{2}-1}\right)}^{\ell}+\|\eta\|_{\widetilde{L}_{t}^{\infty}\left(\dot{B}_{2,1}^{\frac{n}{2}-2}\right)}^{\ell}+\|\mathbb{T}\|_{\widetilde{L}_{t}^{\infty}\left(\dot{B}_{2,1}^{\frac{n}{2}}\right)}^{\ell}+\|(\mathbf{u}, \eta)\|_{\widetilde{L}_{t}^{\infty}\left(\dot{B}_{p, 1}^{\frac{n}{p}-1}\right)}^{h} \\
+\|(a, \mathbb{T})\|_{\widetilde{L}_{t}^{\infty}\left(\dot{B}_{p, 1}^{\frac{n}{p}}\right)}^{h}+\|(a, \mathbf{u})\|_{L_{t}^{1}\left(\dot{B}_{2,1}^{\frac{n}{2}+1}\right)}^{\ell}+\|(\eta, \mathbb{T})\|_{L_{t}^{1}\left(\dot{B}_{2,1}^{\frac{n}{2}}\right)}^{\ell}+\left\|\mathbb{T}^{\ell}\right\|_{L_{t}^{1}\left(\dot{B}_{2,1}^{\frac{n}{2}+2}\right)}+\|(\mathbf{u}, \eta)\|_{L_{t}^{1}\left(\dot{B}_{p, 1}^{\frac{n}{p}+1}\right)}^{h}+\|\mathbb{T}\|_{L_{t}^{1}\left(\dot{B}_{p, 1}^{\frac{n}{p}+2}\right)}^{h} . \\
+\|(a, \mathbb{T})\|_{L_{t}^{1}\left(\dot{B}_{p, 1}^{\frac{n}{p}}\right)}^{h}+\|
\end{gathered}
$$

Remark 1.4. Compared with the result by Wang and Wen [43], the polymer number density is allowed to vanish and the stress tensor can be near zero equilibrium here.

With the global solutions constructed above, next, a natural problem is what is the large time asymptotic behavior of this solutions. The study of the large-time behavior of solutions to the partial different equations is also an old subject. We refer for instance to [18], [44], [47] for the compressible Navier-Stokes equations and [37, [43, [46] for Oldroyd-B model.

One can now state the third result of the present paper.

Theorem 1.5. (Optimal decay) Let $(a, \mathbf{u}, \eta, \mathbb{T})$ be the global small solutions addressed by Theorem 1.3. For any $\frac{n}{2}-\frac{2 n}{p} \leq \sigma<\frac{n}{2}-1$, and $\left(a_{0}^{\ell}, \mathbf{u}_{0}^{\ell}\right) \in \dot{B}_{2, \infty}^{\sigma}\left(\mathbb{R}^{n}\right), \eta_{0}^{\ell} \in \dot{B}_{2, \infty}^{\sigma-1}\left(\mathbb{R}^{n}\right)$, $\mathbb{T}_{0}^{\ell} \in \dot{B}_{2, \infty}^{\sigma+1}\left(\mathbb{R}^{n}\right)$, we have the following time-decay rate

$$
\begin{aligned}
\left\|\Lambda^{\gamma_{1}}(a, \mathbf{u})\right\|_{L^{p}} \leq C(1+t)^{-\frac{n}{2}\left(\frac{1}{2}-\frac{1}{p}\right)-\frac{\gamma_{1}-\sigma}{2}}, & \forall \gamma_{1} \in\left(\frac{n}{p}-\frac{n}{2}+\sigma, \frac{n}{p}-1\right], \\
\left\|\Lambda^{\gamma_{2}} \eta\right\|_{L^{p}} \leq C(1+t)^{-\frac{n}{2}\left(\frac{1}{2}-\frac{1}{p}\right)-\frac{\gamma_{2}-\sigma+1}{2}}, & \forall \gamma_{2} \in\left(\frac{n}{p}-\frac{n}{2}-1+\sigma, \frac{n}{p}-1\right] \\
\left\|\Lambda^{\gamma_{3}} \mathbb{T}\right\|_{L^{p}} \leq C(1+t)^{-\frac{n}{2}\left(\frac{1}{2}-\frac{1}{p}\right)-\frac{\gamma_{3}-\sigma-1}{2}}, & \forall \gamma_{3} \in\left(\frac{n}{p}-\frac{n}{2}+1+\sigma, \frac{n}{p}\right] .
\end{aligned}
$$

Remark 1.6. Let $p=2$, one can deduce from above decay estimates that

$$
\begin{aligned}
& \left\|\Lambda^{\gamma_{1}}(a, \mathbf{u})\right\|_{L^{2}} \leq C(1+t)^{-\frac{\gamma_{1}}{2}+\frac{\sigma}{2}} \\
& \left\|\Lambda^{\gamma_{2}} \eta\right\|_{L^{2}} \leq C(1+t)^{-\frac{\gamma_{2}}{2}+\frac{\sigma-1}{2}} \\
& \left\|\Lambda^{\gamma_{3}} \mathbb{T}\right\|_{L^{2}} \leq C(1+t)^{-\frac{\gamma_{3}}{2}+\frac{\sigma+1}{2}}
\end{aligned}
$$

which coincides with the heat flows, thus our decay rate is optimal in some sense.

Scheme of the proof and organization of the paper. The equations of the polymer number density $\eta$ and the extra stress tensor $\mathbb{T}$ in (1.5) are two heat-type flow, moreover, when the polymer number density $\eta$ and the extra stress tensor $\mathbb{T}$ vanish, the system (1.5) reduces to the barotropic Navier-Stokes equations, thus, we can modify the method used in [16] and [24] to establish the local wellposedness. As the process is lengthy but standard, we only sketch some details in Section 3 to complete the proof of Theorem 1.1.

To prove the Theorem 1.3 regarding the global solutions with small initial data, we use the bootstrap argument, which consists of two main steps. The first step is to establish the $a$ priori bounds while the second is to apply and complete the bootstrap argument by using the a priori bounds. We set the argument in Section 4 which will further be divided into three subsections. Main efforts are devoted to obtaining suitable a priori bounds, and we put it in the first two subsections. To do so, we separate the low frequency from the high frequency to distinguish their different behaviors.

We shall prove the Theorem [1.5] in Section 5. Inspired by the papers [44] and [47], our main task is to establish a Lyapunov-type inequality in time for energy norms (see (5.31) ) by using the pure energy argument (independent of spectral analysis). 
In Section 2, we recall the Littlewood-Paley theory and give some useful lemmas about product laws, commutators estimates in Besov spaces. In the Appendix A, we give some new product laws in Besov spaces.

Let us complete this section by describing the notations which will be used in the sequel. Notations: For two operators $A$ and $B$, we denote $[A, B]=A B-B A$, the commutator between $A$ and $B$. The letter $C$ stands for a generic constant whose meaning is clear from the context. We write $a \lesssim b$ instead of $a \leq C b$. Given a Banach space $X$, we shall denote $\|(a, b)\|_{X}=\|a\|_{X}+\|b\|_{X}$.

For $X$ a Banach space and $I$ an interval of $\mathbb{R}$, we denote by $C(I ; X)$ the set of continuous functions on $I$ with values in $X$, and by $C_{b}(I ; X)$ the subset of bounded functions of $C(I ; X)$. For $q \in[1,+\infty], L^{q}(I ; X)$ stands for the set of measurable functions on $I$ with values in $X$, such that $t \mapsto\|f(t)\|_{X}$ belongs to $L^{q}(I)$. For short, we write $L_{T}^{q}(X)$ instead of $L^{q}((0, T) ; X)$. We always let $\left(d_{j}\right)_{j \in \mathbb{Z}}$ be a generic element of $\ell^{1}(\mathbb{Z})$ so that $\sum_{j \in \mathbb{Z}} d_{j}=1$.

\section{PRELiminaries}

For readers' convenience, in this section, we list some basic knowledge on Littlewood-Paley theory. The Littlewood-Paley decomposition plays a central role in our analysis. To define it, fix some smooth radial non increasing function $\chi$ supported in the ball $B\left(0, \frac{4}{3}\right)$ of $\mathbb{R}^{n}$, and with value 1 on, say, $B\left(0, \frac{3}{4}\right)$, then set $\varphi(\xi)=\chi\left(\frac{\xi}{2}\right)-\chi(\xi)$. We have

$$
\sum_{j \in \mathbb{Z}} \varphi\left(2^{-j} \cdot\right)=1 \text { in } \mathbb{R}^{n} \backslash\{0\} \quad \text { and } \operatorname{Supp} \varphi \subset\left\{\xi \in \mathbb{R}^{n}: \frac{3}{4} \leq|\xi| \leq \frac{8}{3}\right\} \text {. }
$$

The homogeneous dyadic blocks $\dot{\Delta}_{j}$ are defined on tempered distributions by

$$
\dot{\Delta}_{j} u \stackrel{\text { def }}{=} \varphi\left(2^{-j} D\right) u \stackrel{\text { def }}{=} \mathcal{F}^{-1}\left(\varphi\left(2^{-j} \cdot\right) \mathcal{F} u\right) .
$$

Let us remark that, for any homogeneous function $A$ of order 0 smooth outside 0 , we have

$$
\forall p \in[1, \infty], \quad\left\|\dot{\Delta}_{j}(A(D) u)\right\|_{L^{p}} \leq C\left\|\dot{\Delta}_{j} u\right\|_{L^{p}}
$$

Definition 2.1. Let $p, r$ be in $[1,+\infty]$ and $s$ in $\mathbb{R}, u \in \mathcal{S}^{\prime}\left(\mathbb{R}^{n}\right)$. We define the Besov norm by

$$
\|u\|_{\dot{B}_{p, r}^{s}} \stackrel{\text { def }}{=}\left\|\left(2^{j s}\left\|\dot{\Delta}_{j} u\right\|_{L^{p}}\right)_{j}\right\|_{\ell^{r}(\mathbb{Z})} .
$$

We then define the spaces $\dot{B}_{p, r}^{s} \stackrel{\text { def }}{=}\left\{u \in \mathcal{S}_{h}^{\prime}\left(\mathbb{R}^{n}\right), \mid\|u\|_{\dot{B}_{p, r}^{s}}<\infty\right\}$, where $u \in \mathcal{S}_{h}^{\prime}\left(\mathbb{R}^{n}\right)$ means that $u \in \mathcal{S}^{\prime}\left(\mathbb{R}^{n}\right)$ and $\lim _{j \rightarrow-\infty}\left\|\dot{S}_{j} u\right\|_{L^{\infty}}=0$ (see Definition 1.26 of [1]).

When employing parabolic estimates in Besov spaces, it is somehow natural to take the time-Lebesgue norm before performing the summation for computing the Besov norm. So we next introduce the following Besov-Chemin-Lerner space $\widetilde{L}_{T}^{q}\left(\dot{B}_{p, r}^{s}\right)$ (see [1]):

where

$$
\widetilde{L}_{T}^{q}\left(\dot{B}_{p, r}^{s}\right)=\left\{u \in(0,+\infty) \times \mathcal{S}_{h}^{\prime}\left(\mathbb{R}^{n}\right):\|u\|_{\widetilde{L}_{T}^{q}\left(\dot{B}_{p, r}^{s}\right)}<+\infty\right\},
$$

$$
\|u\|_{\widetilde{L}_{T}^{q}\left(\dot{B}_{p, r}^{s}\right)} \stackrel{\text { def }}{=}\left\|2^{k s}\right\| \dot{\Delta}_{k} u(t)\left\|_{L^{q}\left(0, T ; L^{p}\right)}\right\|_{\ell^{r}} .
$$

The index $T$ will be omitted if $T=+\infty$ and we shall denote by $\widetilde{\mathcal{C}}_{b}\left([0, T] ; \dot{B}_{p, r}^{s}\right)$ the subset of functions of $\widetilde{L}_{T}^{\infty}\left(\dot{B}_{p, r}^{s}\right)$ which are also continuous from $[0, T]$ to $\dot{B}_{p, r}^{s}$.

By the Minkowski inequality, we have the following inclusions between the Chemin-Lerner space $\widetilde{L}_{T}^{\lambda}\left(\dot{B}_{p, r}^{s}\right)$ and the Bochner space $L_{T}^{\lambda}\left(\dot{B}_{p, r}^{s}\right)$ :

$$
\|u\|_{\tilde{L}_{T}^{\lambda}\left(\dot{B}_{p, r}^{s}\right)} \leq\|u\|_{L_{T}^{\lambda}\left(\dot{B}_{p, r}^{s}\right)} \quad \text { if } \lambda \leq r, \quad\|u\|_{\widetilde{L}_{T}^{\lambda}\left(\dot{B}_{p, r}^{s}\right)} \geq\|u\|_{L_{T}^{\lambda}\left(\dot{B}_{p, r}^{s}\right)}, \quad \text { if } \lambda \geq r .
$$


The following Bernstein's lemma will be repeatedly used throughout this paper.

Lemma 2.2. Let $\mathcal{B}$ be a ball and $\mathcal{C}$ a ring of $\mathbb{R}^{n}$. A constant $C$ exists so that for any positive real number $\lambda$, any non-negative integer $k$, any smooth homogeneous function $\sigma$ of degree $m$, and any couple of real numbers $(p, q)$ with $1 \leq p \leq q \leq \infty$, there hold

$$
\begin{array}{r}
\operatorname{Supp} \hat{u} \subset \lambda \mathcal{B} \Rightarrow \sup _{|\alpha|=k}\left\|\partial^{\alpha} u\right\|_{L^{q}} \leq C^{k+1} \lambda^{k+n\left(\frac{1}{p}-\frac{1}{q}\right)}\|u\|_{L^{p}}, \\
\operatorname{Supp} \hat{u} \subset \lambda \mathcal{C} \Rightarrow C^{-k-1} \lambda^{k}\|u\|_{L^{p}} \leq \sup _{|\alpha|=k}\left\|\partial^{\alpha} u\right\|_{L^{p}} \leq C^{k+1} \lambda^{k}\|u\|_{L^{p}}, \\
\operatorname{Supp} \hat{u} \subset \lambda \mathcal{C} \Rightarrow\|\sigma(D) u\|_{L^{q}} \leq C_{\sigma, m} \lambda^{m+n\left(\frac{1}{p}-\frac{1}{q}\right)}\|u\|_{L^{p}} .
\end{array}
$$

Next we recall a few nonlinear estimates in Besov spaces which may be obtained by means of paradifferential calculus. Here, we recall the decomposition in the homogeneous context:

$$
u v=\dot{T}_{u} v+\dot{T}_{v} u+\dot{R}(u, v)=\dot{T}_{u} v+\dot{T}_{v}^{\prime} u
$$

where

$$
\dot{T}_{u} v \stackrel{\text { def }}{=} \sum_{j \in \mathbb{Z}} \dot{S}_{j-1} u \dot{\Delta}_{j} v, \quad \dot{R}(u, v) \stackrel{\text { def }}{=} \sum_{j \in Z} \dot{\Delta}_{j} u \tilde{\dot{\Delta}}_{j} v
$$

and

$$
\tilde{\dot{\Delta}}_{j} v \stackrel{\text { def }}{=} \sum_{\left|j-j^{\prime}\right| \leq 1} \dot{\Delta}_{j^{\prime}} v, \quad \dot{T}_{v}^{\prime} u \stackrel{\text { def }}{=} \sum_{j \in Z} \dot{S}_{j+2} v \dot{\Delta}_{j} u .
$$

The paraproduct $\dot{T}$ and the remainder $\dot{R}$ operators satisfy the following continuous properties.

Lemma $2.4([1])$. For all $s \in \mathbb{R}, \sigma \geq 0$, and $1 \leq p, p_{1}, p_{2} \leq \infty$, the paraproduct $\dot{T}$ is a bilinear, continuous operator from $\dot{B}_{p_{1}, 1}^{-\sigma} \times \dot{B}_{p_{2}, 1}^{s}$ to $\dot{B}_{p, 1}^{s-\sigma}$ with $\frac{1}{p}=\frac{1}{p_{1}}+\frac{1}{p_{2}}$. The remainder $\dot{R}$ is bilinear continuous from $\dot{B}_{p_{1}, 1}^{s_{1}} \times \dot{B}_{p_{2}, 1}^{s_{2}}$ to $\dot{B}_{p, 1}^{s_{1}+s_{2}}$ with $s_{1}+s_{2}>0$, and $\frac{1}{p}=\frac{1}{p_{1}}+\frac{1}{p_{2}}$.

Lemma 2.5. ([18, Proposition A.1]) Let $1 \leq p, q \leq \infty, s_{1} \leq \frac{n}{q}, s_{2} \leq n \min \left\{\frac{1}{p}, \frac{1}{q}\right\}$ and $s_{1}+s_{2}>n \max \left\{0, \frac{1}{p}+\frac{1}{q}-1\right\}$. For $\forall(u, v) \in \dot{B}_{q, 1}^{s_{1}}\left(\mathbb{R}^{n}\right) \times \dot{B}_{p, 1}^{s_{2}}\left(\mathbb{R}^{n}\right)$, we have

$$
\|u v\|_{\dot{B}_{p, 1}^{s_{1}+s_{2}-\frac{n}{q}}} \lesssim\|u\|_{\dot{B}_{q, 1}^{s_{1}}}\|v\|_{\dot{B}_{p, 1}^{s_{2}}} .
$$

Lemma 2.6. Let $n \geq 2$ and $2 \leq p \leq \min (4,2 n /(n-2))$ and, additionally, $p \neq 4$ if $n=2$. For any $u \in \dot{B}_{p, 1}^{\frac{n}{p}}\left(\mathbb{R}^{n}\right), v^{\ell} \in \dot{B}_{2,1}^{\frac{n}{2}-1}\left(\mathbb{R}^{n}\right)$ and $v^{h} \in \dot{B}_{p, 1}^{\frac{n}{p}-1}\left(\mathbb{R}^{n}\right)$, we have

$$
\left\|(u v)^{\ell}\right\|_{\dot{B}_{2,1}^{\frac{n}{2}-1}} \lesssim\left(\left\|v^{\ell}\right\|_{\dot{B}_{2,1}^{\frac{n}{2}-1}}+\left\|v^{h}\right\|_{\dot{B}_{p, 1}^{\frac{n}{p}-1}}\right)\|u\|_{\dot{B}_{p, 1}^{\frac{n}{p}}}
$$

Proof. We first use Bony's decomposition to write

$$
\dot{S}_{j_{0}+1}(u v)=\dot{T}_{u} \dot{S}_{j_{0}+1} v+\dot{S}_{j_{0}+1}\left(\dot{T}_{v} u+\dot{R}(v, u)\right)+\left[\dot{S}_{j_{0}+1}, \dot{T}_{u}\right] v .
$$

Applying Lemma 2.4, we have

$$
\left\|\dot{T}_{u} \dot{S}_{j_{0}+1} v\right\|_{\dot{B}_{2,1}^{\frac{n}{2}-1}} \lesssim\|u\|_{L^{\infty}}\left\|v^{\ell}\right\|_{\dot{B}_{2,1}^{\frac{n}{2}-1}} \lesssim\left\|v^{\ell}\right\|_{\dot{B}_{2,1}^{\frac{n}{2}-1}}\|u\|_{\dot{B}_{p, 1}^{\frac{n}{p}}}
$$

and $\left(\frac{1}{p *}=\frac{1}{2}-\frac{1}{p}\right)$

$$
\left\|\dot{S}_{j_{0}+1}\left(\dot{T}_{v} u+\dot{R}(v, u)\right)\right\|_{\dot{B}_{2,1}^{\frac{n}{2}-1}} \lesssim\|v\|_{\dot{B}_{p^{*}, 1}^{\frac{n}{p^{*}}-1}}\|u\|_{\dot{B}_{p, 1}^{\frac{n}{p}}} \lesssim\|v\|_{\dot{B}_{p, 1}^{\frac{n}{p}-1}}\|u\|_{\dot{B}_{p, 1}^{\frac{n}{p}}}
$$


By Lemma 6.1 in [17], the term about the commutator can be bounded

$$
\left\|\left[\dot{S}_{j_{0}+1}, \dot{T}_{u}\right] v\right\|_{\dot{B}_{2,1}^{\frac{n}{2}-1}} \lesssim\|\nabla u\|_{\dot{B}_{p^{*}, 1}^{\frac{n}{p^{*}}-1}}\|v\|_{\dot{B}_{p, 1}^{\frac{n}{p}-1}} \lesssim\|v\|_{\dot{B}_{p, 1}^{\frac{n}{p}-1}}\|u\|_{\dot{B}_{p, 1}^{\frac{n}{p}}}
$$

Thus, the combination of (2.8) $-(2.10)$ shows the validity of (2.7).

We also need the following Classical commutator's estimate:

Lemma 2.11. ([1], Lemma 2.100]) Let $1 \leq p \leq \infty,-n \min \left\{\frac{1}{p}, 1-\frac{1}{p}\right\}<s \leq \frac{n}{p}$. For any $v \in \dot{B}_{p, 1}^{s}\left(\mathbb{R}^{n}\right)$ and $\nabla u \in \dot{B}_{p, 1}^{\frac{n}{p}}\left(\mathbb{R}^{n}\right)$, there holds

$$
\left\|\left[\dot{\Delta}_{j}, u \cdot \nabla\right] v\right\|_{L^{p}} \lesssim d_{j} 2^{-j s}\|\nabla u\|_{\dot{B}_{p, 1}^{\frac{n}{p}}\|v\|_{\dot{B}_{p, 1}^{s}}}
$$

Finally, we recall a composition result and the parabolic regularity estimate for the heat equation to end this section.

Lemma 2.12. ([1]) Let $G$ with $G(0)=0$ be a smooth function defined on an open interval $I$ of $\mathbb{R}$ containing 0 . Then the following estimates

$$
\|G(a)\|_{\dot{B}_{p, 1}^{s}} \lesssim\|a\|_{\dot{B}_{p, 1}^{s}} \quad \text { and } \quad\|G(a)\|_{\widetilde{L}_{T}^{q}\left(\dot{B}_{p, 1}^{s}\right)} \lesssim\|a\|_{\widetilde{L}_{T}^{q}\left(\dot{B}_{p, 1}^{s}\right)}
$$

hold true for $s>0,1 \leq p, q \leq \infty$ and a valued in a bounded interval $J \subset I$.

Lemma $2.13([1])$. Let $\sigma \in \mathbb{R}, T>0,1 \leq p, r \leq \infty$ and $1 \leq q_{2} \leq q_{1} \leq \infty$. Let $u$ satisfy the heat equation

$$
\partial_{t} u-\Delta u=f, \quad u_{\mid t=0}=u_{0} .
$$

Then there holds the following a priori estimate

$$
\|u\|_{\widetilde{L}_{T}^{q_{1}\left(\dot{B}_{p, r}^{\left.\sigma+\frac{2}{q_{1}}\right)}\right.}} \lesssim\left\|u_{0}\right\|_{\dot{B}_{p, r}^{\sigma}}+\|f\|_{\widetilde{L}_{T}^{q_{2}\left(\dot{B}_{p, r}^{\left.\sigma-2+\frac{2}{q_{2}}\right)}\right.}} .
$$

\section{The proof of Theorem 1.1}

In order to prove the existence part of Theorem 1.1, we use a scheme similar to the case of barotropic Navier-Stokes equations, see [12, [16], 24] for example. More precisely, let

$$
\mathbf{u}_{\mathfrak{F}}=e^{t \mathcal{A}} \mathbf{u}_{0} \quad \text { with } \quad \mathcal{A} \stackrel{\text { def }}{=} \mu \Delta+(\lambda+\mu) \nabla \operatorname{div}, \quad \eta_{\mathfrak{F}}=e^{\varepsilon t \Delta} \eta_{0}
$$

and $\mathbb{T}_{\mathfrak{F}}$ be the solution to the linear system

$$
\partial_{t} \mathbb{T}_{\mathfrak{F}}+\frac{A_{0}}{2 \lambda_{1}} \mathbb{T}_{\mathfrak{F}}-\varepsilon \Delta \mathbb{T}_{\mathfrak{F}}=0, \quad \mathbb{T}_{\mathfrak{F}}(0)=\mathbb{T}_{0}
$$

Denote $\overline{\mathbf{u}}=\mathbf{u}-\mathbf{u}_{\mathfrak{F}}, \quad \bar{\eta}=\eta-\eta_{\mathfrak{F}}, \quad \overline{\mathbb{T}}=\mathbb{T}-\mathbb{T}_{\mathfrak{F}}$, then $(a, \overline{\mathbf{u}}, \bar{\eta}, \overline{\mathbb{T}})$ satisfies the following equations:

$$
\left\{\begin{array}{l}
\partial_{t} a+\operatorname{div} \mathbf{u}=-\operatorname{div}(a \mathbf{u}), \\
\partial_{t} \bar{\eta}-\varepsilon \Delta \bar{\eta}=-\operatorname{div}(\eta \mathbf{u}), \\
\partial_{t} \overline{\mathbb{T}}+\frac{A_{0}}{2 \lambda_{1}} \overline{\mathbb{T}}+(\mathbf{u} \cdot \nabla) \mathbb{T}-\varepsilon \Delta \overline{\mathbb{T}}=\frac{\kappa A_{0}}{2 \lambda_{1}} \eta \mathbb{I}+F(\mathbb{T}, \mathbf{u}), \\
\partial_{t} \overline{\mathbf{u}}+\mathbf{u} \cdot \nabla \mathbf{u}-\mu \Delta \overline{\mathbf{u}}-(\lambda+\mu) \nabla \operatorname{div} \overline{\mathbf{u}}+\nabla a=\operatorname{div} \mathbb{T}-\kappa L \nabla \eta+G(a, \mathbf{u}, \eta, \mathbb{T}) .
\end{array}\right.
$$

We use a standard scheme for proving the existence of the solutions.

- We smooth out the data and get a sequence of smooth solutions $\left(a^{n}, \overline{\mathbf{u}}^{n}, \bar{\eta}^{n}, \overline{\mathbb{T}}^{n}\right)$ of an approximated system of (3.1), on a bounded interval $\left[0 ; T^{n}\right]$ which may depend on $n$.

- We exhibit a positive lower bound $T$ for $T^{n}$, and prove uniform estimates on $\left(a^{n}, \overline{\mathbf{u}}^{n}\right.$, $\left.\bar{\eta}^{n}, \overline{\mathbb{T}}^{n}\right)$. 
- We use compactness to prove that the sequence $\left(a^{n}, \overline{\mathbf{u}}^{n}, \bar{\eta}^{n}, \overline{\mathbb{T}}^{n}\right)$ converges, up to extraction, to a solution of (3.1).

Since the proof is lengthy but standard, we refer for instance to [1], [12, [17, [24, here, we omit the details for brevity. Moreover, the uniqueness can be obtained in the same way as [16].

\section{The PRoOF of TheOREM 1.3}

This section presents the proof of Theorem 1.3 stating the global wellposedness for (1.5). The framework is the bootstrap argument, which consists of two main steps. The first step is to establish the a priori bounds while the second is to apply and complete the bootstrap argument by using the a priori bounds. Main efforts are devoted to obtaining suitable a priori bounds. To do so, we separate the low frequency from the high frequency to distinguish their different behaviors. We make use of some sharp commutator estimates to shift derivatives.

4.1. The estimates in the low frequency. The goal of this subsection is to establish the $a$ priori bounds in the low frequency part, which will be divided into the following three lemmas.

In the first lemma, we are concerned with the estimates about the polymer number density.

Lemma 4.1. Under the conditions in Theorem 1.3, there holds

$$
\begin{aligned}
& \left\|\eta^{\ell}\right\|_{\widetilde{L}_{t}^{\infty}\left(\dot{B}_{2,1}^{\frac{n}{2}-2}\right)}+\varepsilon\left\|\eta^{\ell}\right\|_{L_{t}^{1}\left(\dot{B}_{2,1}^{\frac{n}{2}}\right)} \\
& \quad \lesssim\left\|\eta_{0}^{\ell}\right\|_{\dot{B}_{2,1}^{\frac{n}{2}-2}}+\int_{0}^{t}\left(\left\|\eta^{\ell}\right\|_{\dot{B}_{2,1}^{\frac{n}{2}}}+\left\|\eta^{h}\right\|_{\dot{B}_{p, 1}^{\frac{n}{p}}}\right)\left(\left\|\mathbf{u}^{\ell}\right\|_{\dot{B}_{2,1}^{\frac{n}{2}-1}}+\left\|\mathbf{u}^{h}\right\|_{\dot{B}_{p, 1}^{\frac{n}{p}-1}}\right) d s .
\end{aligned}
$$

Proof. Applying $\dot{\Delta}_{j}$ to the second equation of (1.5), then taking the $L^{2}$ inner product with $\dot{\Delta}_{j} \eta$, we get

$$
\frac{1}{2} \frac{d}{d t}\left\|\dot{\Delta}_{j} \eta\right\|_{L^{2}}^{2}+c \varepsilon 2^{2 j}\left\|\dot{\Delta}_{j} \eta\right\|_{L^{2}}^{2} \lesssim\left\|\dot{\Delta}_{j} \operatorname{div}(\eta \mathbf{u})\right\|_{L^{2}}\left\|\dot{\Delta}_{j} \eta\right\|_{L^{2}}
$$

in which we have used the following Bernstein's inequality: there exists a positive constant $c$ so that

$$
-\int_{\mathbb{R}^{n}} \Delta \dot{\Delta}_{j} \eta \cdot \dot{\Delta}_{j} \eta d x \geq c 2^{2 j}\left\|\dot{\Delta}_{j} \eta\right\|_{L^{2}}^{2} .
$$

Multiplying by $1 /\left\|\dot{\Delta}_{j} \eta\right\|_{L^{2}} 2^{j\left(\frac{n}{2}-2\right)}$ formally on both hand side of (4.3), integrating the resultant inequality from 0 to $t$, we can get by summing up about $j \leq j_{0}$ that

$$
\begin{aligned}
\left\|\eta^{\ell}\right\|_{\widetilde{L}_{t}^{\infty}\left(\dot{B}_{2,1}^{\frac{n}{2}-2}\right)}+\varepsilon\left\|\eta^{\ell}\right\|_{L_{t}^{1}\left(\dot{B}_{2,1}^{\frac{n}{2}}\right)} & \lesssim\left\|\eta_{0}^{\ell}\right\|_{\dot{B}_{2,1}^{\frac{n}{2}-2}}+\int_{0}^{t}\left\|(\operatorname{div}(\eta \mathbf{u}))^{\ell}\right\|_{\dot{B}_{2,1}^{\frac{n}{2}-2}} d s \\
& \lesssim\left\|\eta_{0}^{\ell}\right\|_{\dot{B}_{2,1}^{\frac{n}{2}-2}}+\int_{0}^{t}\left\|(\eta \mathbf{u})^{\ell}\right\|_{\dot{B}_{2,1}^{\frac{n}{2}-1}} d s .
\end{aligned}
$$

It follows from Lemma 2.6 and the embedding relation that

$$
\begin{aligned}
\left\|(\eta \mathbf{u})^{\ell}\right\|_{\dot{B}_{2,1}^{\frac{n}{2}-1}} & \lesssim\|\eta\|_{\dot{B}_{p, 1}^{\frac{n}{p}}}\left(\left\|\mathbf{u}^{\ell}\right\|_{\dot{B}_{2,1}^{\frac{n}{2}-1}}+\left\|\mathbf{u}^{h}\right\|_{\dot{B}_{p, 1}^{\frac{n}{p}-1}}\right) \\
& \lesssim\left(\left\|\eta^{\ell}\right\|_{\dot{B}_{2,1}^{\frac{n}{2}}}+\left\|\eta^{h}\right\|_{\dot{B}_{p, 1}^{\frac{n}{p}}}\right)\left(\left\|\mathbf{u}^{\ell}\right\|_{\dot{B}_{2,1}^{\frac{n}{2}-1}}+\left\|\mathbf{u}^{h}\right\|_{\dot{B}_{p, 1}^{\frac{n}{p}-1}}\right) .
\end{aligned}
$$

This together with (4.4) gives (4.2). 
Lemma 4.6. Under the conditions in Theorem 1.3, there holds

$$
\begin{aligned}
& \left\|\mathbb{T}^{\ell}\right\|_{\widetilde{L}_{t}^{\infty}\left(\dot{B}_{2,1}^{\frac{n}{2}}\right)}+\frac{A_{0}}{2 \lambda_{1}}\left\|\mathbb{T}^{\ell}\right\|_{L_{t}^{1}\left(\dot{B}_{2,1}^{\frac{n}{2}}\right)}+\varepsilon\left\|\mathbb{T}^{\ell}\right\|_{L_{t}^{1}\left(\dot{B}_{2,1}^{\frac{n}{2}+2}\right)} \\
& \lesssim\left\|\mathbb{T}_{0}^{\ell}\right\|_{\dot{B}_{2,1}^{\frac{n}{2}}}+\frac{\kappa A_{0}}{2 \lambda_{1}} \int_{0}^{t}\left\|\eta^{\ell}\right\|_{\dot{B}_{2,1}^{\frac{n}{2}}} d s+\int_{0}^{t}\left(\left\|\mathbb{T}^{\ell}\right\|_{\dot{B}_{2,1}^{\frac{n}{2}}}^{2}+\left\|\mathbb{T}^{h}\right\|_{\dot{B}_{p, 1}^{\frac{n}{p}}}^{2}\right) d s \\
& \quad+\int_{0}^{t}\left(\left\|\mathbf{u}^{\ell}\right\|_{\dot{B}_{2,1}^{\frac{n}{2}-1}}+\left\|\mathbf{u}^{h}\right\|_{\dot{B}_{p, 1}^{\frac{n}{p}-1}}\right)\left(\left\|\mathbf{u}^{\ell}\right\|_{\dot{B}_{2,1}^{\frac{n}{2}+1}}+\left\|\mathbf{u}^{h}\right\|_{\dot{B}_{p, 1}^{\frac{n}{p}+1}}\right) d s .
\end{aligned}
$$

Proof. Along the same derivation of (4.4), we can deduce from the third equation of (1.5) that

$$
\begin{aligned}
& \left\|\mathbb{T}^{\ell}\right\|_{\widetilde{L}_{t}^{\infty}\left(\dot{B}_{2,1}^{2}\right)}+\frac{A_{0}}{2 \lambda_{1}}\left\|\mathbb{T}^{\ell}\right\|_{L_{t}^{1}\left(\dot{B}_{2,1}^{\frac{n}{2}}\right)}+\varepsilon\left\|\mathbb{T}^{\ell}\right\|_{L_{t}^{1}\left(\dot{B}_{2,1}^{\frac{n}{2}+2}\right)} \\
& \quad \lesssim\left\|\mathbb{T}_{0}^{\ell}\right\|_{\dot{B}_{2,1}^{\frac{n}{2}}}+\frac{\kappa A_{0}}{2 \lambda_{1}} \int_{0}^{t}\left\|\eta^{\ell}\right\|_{\dot{B}_{2,1}^{\frac{n}{2}}} d s+\int_{0}^{t}\left\|(F(\mathbb{T}, \mathbf{u}))^{\ell}\right\|_{\dot{B}_{2,1}^{\frac{n}{2}}} d s+\int_{0}^{t}\left\|(\mathbf{u} \cdot \nabla \mathbb{T})^{\ell}\right\|_{\dot{B}_{2,1}^{\frac{n}{2}}} d s .
\end{aligned}
$$

Applying Lemma 2.6 once again, we have

$$
\begin{aligned}
& \left\|(F(\mathbb{T}, \mathbf{u}))^{\ell}\right\|_{\dot{B}_{2,1}^{\frac{n}{2}}}+\left\|(\mathbf{u} \cdot \nabla \mathbb{T})^{\ell}\right\|_{\dot{B}_{2,1}^{\frac{n}{2}-1}} \\
& \quad \lesssim\left\|(F(\mathbb{T}, \mathbf{u}))^{\ell}\right\|_{\dot{B}_{2,1}^{\frac{n}{2}-1}}+\left\|(\mathbf{u} \cdot \nabla \mathbb{T})^{\ell}\right\|_{\dot{B}_{2,1}^{\frac{n}{2}-1}} \\
& \quad \lesssim\|\mathbb{T}\|_{\dot{B}_{p, 1}^{\frac{n}{p}}}\left(\left\|\nabla \mathbf{u}^{\ell}\right\|_{\dot{B}_{2,1}^{\frac{n}{2}-1}}+\left\|\nabla \mathbf{u}^{h}\right\|_{\dot{B}_{p, 1}^{\frac{n}{p}-1}}\right)+\|\mathbf{u}\|_{\dot{B}_{p, 1}^{\frac{n}{p}}}\left(\left\|\nabla \mathbb{T}^{\ell}\right\|_{\dot{B}_{2,1}^{\frac{n}{2}-1}}+\left\|\nabla \mathbb{T}^{h}\right\|_{\dot{B}_{p, 1}^{\frac{n}{p}-1}}\right) \\
& \quad \lesssim\|\mathbb{T}\|_{\dot{B}_{p, 1}^{\frac{n}{p}}}\left(\mathbf{u}^{\ell}\left\|_{\dot{B}_{2,1}^{\frac{n}{2}}}+\right\| \mathbf{u}^{h} \|_{\dot{B}_{p, 1}^{\frac{n}{p}}}\right)+\|\mathbf{u}\|_{\dot{B}_{p, 1}^{\frac{n}{p}}}\left(\left\|\mathbb{T}^{\ell}\right\|_{\dot{B}_{2,1}^{\frac{n}{2}}}+\left\|\mathbb{T}^{h}\right\|_{\dot{B}_{p, 1}^{\frac{n}{p}}}\right) \\
& \quad \lesssim\left\|\mathbb{T}^{\ell}\right\|_{\dot{B}_{2,1}^{\frac{n}{2}}}^{2}+\left\|\mathbb{T}^{h}\right\|_{\dot{B}_{p, 1}^{\frac{n}{p}}}^{2}+\left(\left\|\mathbf{u}^{\ell}\right\|_{\dot{B}_{2,1}^{\frac{n}{2}-1}}+\left\|\mathbf{u}^{h}\right\|_{\dot{B}_{p, 1}^{\frac{n}{p}-1}}\right)\left(\left\|\mathbf{u}^{\ell}\right\|_{\dot{B}_{2,1}^{\frac{n}{2}+1}}+\left\|\mathbf{u}^{h}\right\|_{\dot{B}_{p, 1}^{\frac{n}{p}+1}}\right) .
\end{aligned}
$$

Inserting the above estimate into (4.8) implies (4.7).

Lemma 4.9. Under the conditions in Theorem 1.3, there holds

$$
\begin{aligned}
& \left\|\left(a^{\ell}, \mathbf{u}^{\ell}\right)\right\|_{\widetilde{L}_{t}^{\infty}\left(\dot{B}_{2,1}^{\frac{n}{2}-1}\right)}+\mu\left\|\left(a^{\ell}, \mathbf{u}^{\ell}\right)\right\|_{L_{t}^{1}\left(\dot{B}_{2,1}^{\frac{n}{2}+1}\right)} \\
& \quad \lesssim\left\|\left(a_{0}^{\ell}, \mathbf{u}_{0}^{\ell}\right)\right\|_{\dot{B}_{2,1}^{\frac{n}{2}-1}}+\|\mathbb{T}\|_{L_{t}^{1}\left(\dot{B}_{2,1}^{\frac{n}{2}}\right)}^{\ell}+\kappa L\|\eta\|_{L_{t}^{1}\left(\dot{B}_{2,1}^{\frac{n}{2}}\right)}^{\ell}+\int_{0}^{t} \mathcal{N}_{1}(s) d s,
\end{aligned}
$$

with

$$
\begin{gathered}
\mathcal{N}_{1}(t) \stackrel{\text { def }}{=}\left(\left\|\left(a^{\ell}, \mathbf{u}^{\ell}\right)\right\|_{\dot{B}_{2,1}^{\frac{n}{2}-1}}+\left\|\mathbf{u}^{h}\right\|_{\dot{B}_{p, 1}^{\frac{n}{p}-1}}\right)\left(\left\|\left(a^{\ell}, \mathbf{u}^{\ell}\right)\right\|_{\dot{B}_{2,1}^{\frac{n}{2}+1}}+\left\|\mathbf{u}^{h}\right\|_{\dot{B}_{p, 1}^{\frac{n}{p}+1}}+\left\|\eta^{\ell}\right\|_{\dot{B}_{2,1}^{\frac{n}{2}}}+\left\|\eta^{h}\right\|_{\dot{B}_{p, 1}^{\frac{n}{p}}}\right) \\
+\left(\left\|a^{\ell}\right\|_{\dot{B}_{2,1}^{\frac{n}{2}-1}}+\left\|\mathbb{T}^{\ell}\right\|_{\dot{B}_{2,1}^{\frac{n}{2}}}+\left\|\left(a^{h}, \mathbb{T}^{h}\right)\right\|_{\dot{B}_{p, 1}^{\frac{n}{p}}}\right)\left(\left\|\left(\eta^{\ell}, \mathbb{T}^{\ell}\right)\right\|_{\dot{B}_{2,1}^{\frac{n}{2}}}+\left\|\left(a^{h}, \mathbb{T}^{h}\right)\right\|_{\dot{B}_{p, 1}^{\frac{n}{p}}}\right. \\
\left.+\left\|\eta^{h}\right\|_{\dot{B}_{p, 1}^{\frac{n}{p}+1}}+\left\|\eta^{\ell}\right\|_{\dot{B}_{2,1}^{\frac{n}{2}-2}}\left\|\eta^{\ell}\right\|_{\dot{B}_{2,1}^{\frac{n}{2}}}+\left\|\eta^{h}\right\|_{\dot{B}_{p, 1}^{\frac{n}{p}-1}}\left\|\eta^{h}\right\|_{\dot{B}_{p, 1}^{\frac{n}{p}+1}}\right) .
\end{gathered}
$$

Proof. The combination of the first equation and the fourth equation in (1.5) is similar to the compressible Navier-Stokes equation up to some nonlinear terms, thus, by using the operators $\mathcal{P}$ and $\mathcal{Q}$, we get the equation of incompressible part

$$
\partial_{t} \mathcal{P} \mathbf{u}-\mu \Delta \mathcal{P} \mathbf{u}=-\mathcal{P}(\mathbf{u} \cdot \nabla \mathbf{u})+\mathcal{P} \operatorname{div} \mathbb{T}+\mathcal{P} G(a, \mathbf{u}, \eta, \mathbb{T}),
$$

and the equation of compressible part

$$
\left\{\begin{array}{l}
\partial_{t} a+\operatorname{div} \mathbf{u}=-\operatorname{div}(a \mathbf{u}), \\
\partial_{t} \mathcal{Q} \mathbf{u}-\nu \Delta \mathcal{Q} \mathbf{u}+\nabla a=-\mathcal{Q}(\mathbf{u} \cdot \nabla \mathbf{u})+\mathcal{Q} \operatorname{div} \mathbb{T}-\kappa L \nabla \eta+\mathcal{Q} G(a, \mathbf{u}, \eta, \mathbb{T}) .
\end{array}\right.
$$


Applying $\dot{\Delta}_{j}$ to (4.12) and taking $L^{2}$ inner product of the resulting equation with $\dot{\Delta}_{j} \mathcal{P} \mathbf{u}$, applying the Hölder inequality and integrating the resultant inequality over $[0, t]$, then multiplying the inequality by $2^{\left(\frac{n}{2}-1\right) j}$ and taking summation for $j \leq j_{0}$, we arrive at

$$
\begin{aligned}
\left\|\mathcal{P} \mathbf{u}^{\ell}\right\|_{\widetilde{L}_{t}^{\infty}\left(\dot{B}_{2,1}^{\frac{n}{2}-1}\right)}+ & \mu\left\|\mathcal{P} \mathbf{u}^{\ell}\right\|_{L_{t}^{1}\left(\dot{B}_{2,1}^{\frac{n}{2}+1}\right)} \lesssim\left\|\mathcal{P} \mathbf{u}_{0}^{\ell}\right\|_{\dot{B}_{2,1}^{\frac{n}{2}-1}}+\left\|(\mathcal{P} \operatorname{div} \mathbb{T})^{\ell}\right\|_{L_{t}^{1}\left(\dot{B}_{2,1}^{\frac{n}{2}-1}\right)} \\
& +\left\|(\mathcal{P}(\mathbf{u} \cdot \nabla \mathbf{u}))^{\ell}\right\|_{L_{t}^{1}\left(\dot{B}_{2,1}^{\frac{n}{2}-1}\right)}+\left\|(\mathcal{P} G(a, \mathbf{u}, \eta, \mathbb{T}))^{\ell}\right\|_{L_{t}^{1}\left(\dot{B}_{2,1}^{\frac{n}{2}-1}\right)}
\end{aligned}
$$

For the equations in (4.13), an energy estimates for the barotropic linearized equations (see [1], Prop. 10.23, or [15]) thus give

$$
\begin{aligned}
& \left\|(a, \mathcal{Q} \mathbf{u})^{\ell}\right\|_{\widetilde{L}_{t}^{\infty}\left(\dot{B}_{2,1}^{\frac{n}{2}-1}\right)}+\nu\left\|(a, \mathcal{Q} \mathbf{u})^{\ell}\right\|_{L_{t}^{1}\left(\dot{B}_{2,1}^{\frac{n}{2}+1}\right)} \\
& \lesssim\left\|\left(a_{0}, \mathcal{Q} \mathbf{u}_{0}\right)^{\ell}\right\|_{\dot{B}_{2,1}^{\frac{n}{2}-1}}+\int_{0}^{t}\left\|(\operatorname{div} \mathbb{T})^{\ell}\right\|_{\dot{B}_{2,1}^{\frac{n}{2}-1}} d s+\kappa L \int_{0}^{t}\left\|(\nabla \eta)^{\ell}\right\|_{\dot{B}_{2,1}^{\frac{n}{2}-1}} d s \\
& +\int_{0}^{t}\left\|(\operatorname{div}(a \mathbf{u}))^{\ell}\right\|_{\dot{B}_{2,1}^{\frac{n}{2}-1}} d s+\int_{0}^{t}\left\|(\mathbf{u} \cdot \nabla \mathbf{u})^{\ell}\right\|_{\dot{B}_{2,1}^{\frac{n}{2}-1}} d s+\int_{0}^{t}\left\|(G(a, \mathbf{u}, \eta, \mathbb{T}))^{\ell}\right\|_{\dot{B}_{2,1}^{\frac{n}{2}-1}} d s .
\end{aligned}
$$

Summing up the above two estimates implies that

$$
\begin{aligned}
& \left\|\left(a^{\ell}, \mathbf{u}^{\ell}\right)\right\|_{\widetilde{L}_{t}^{\infty}\left(\dot{B}_{2,1}^{\frac{n}{2}-1}\right)}+\mu\left\|\left(a^{\ell}, \mathbf{u}^{\ell}\right)\right\|_{L_{t}^{1}\left(\dot{B}_{2,1}^{\frac{n}{2}+1}\right)} \\
& \quad \lesssim\left\|\left(a_{0}^{\ell}, \mathbf{u}_{0}^{\ell}\right)\right\|_{\dot{B}_{2,1}^{\frac{n}{2}-1}}+\|\mathbb{T}\|_{L_{t}^{1}\left(\dot{B}_{2,1}^{\frac{n}{2}}\right)}^{\ell}+\kappa L\|\eta\|_{L_{t}^{1}\left(\dot{B}_{2,1}^{2}\right)}^{\ell} \\
& \quad+\|(\mathbf{u} \cdot \nabla \mathbf{u})\|_{L_{t}^{1}\left(\dot{B}_{2,1}^{\frac{n}{2}}-1\right)}^{\ell}+\|\operatorname{div}(a \mathbf{u})\|_{L_{t}^{1}\left(\dot{B}_{2,1}^{\frac{n}{2}-1}\right)}^{\ell}+\|G(a, \mathbf{u}, \eta, \mathbb{T})\|_{L_{t}^{1}\left(\dot{B}_{2,1}^{\frac{n}{2}}-1\right.}^{\ell} \cdot
\end{aligned}
$$

Next, we deal with nonlinear terms on the right hand side of (4.15).

At first, thanks to Lemma 2.6, one has

$$
\begin{aligned}
\left\|(\mathbf{u} \cdot \nabla \mathbf{u})^{\ell}\right\|_{\dot{B}_{2,1}^{\frac{n}{2}-1}} & \lesssim\left(\left\|\mathbf{u}^{\ell}\right\|_{\dot{B}_{2,1}^{\frac{n}{2}-1}}+\left\|\mathbf{u}^{h}\right\|_{\dot{B}_{p, 1}^{\frac{n}{p}-1}}\right)\|\nabla \mathbf{u}\|_{\dot{B}_{p, 1}^{\frac{n}{p}}} \\
& \lesssim\left(\left\|\mathbf{u}^{\ell}\right\|_{\dot{B}_{2,1}^{\frac{n}{2}-1}}+\left\|\mathbf{u}^{h}\right\|_{\dot{B}_{p, 1}^{\frac{n}{p}-1}}\right)\left(\left\|\mathbf{u}^{\ell}\right\|_{\dot{B}_{2,1}^{\frac{n}{2}+1}}+\left\|\mathbf{u}^{h}\right\|_{\dot{B}_{p, 1}^{\frac{n}{p}+1}}\right) .
\end{aligned}
$$

Similarly, from Lemmas 2.6 and 2.12, there hold the following three estimates:

$$
\begin{aligned}
\left\|(\operatorname{div}(a \mathbf{u}))^{\ell}\right\|_{\dot{B}_{2,1}^{\frac{n}{2}-1}} & \lesssim\|\mathbf{u}\|_{\dot{B}_{p, 1}^{\frac{n}{p}}}\left(\left\|a^{h}\right\|_{\dot{B}_{p, 1}^{\frac{n}{p}}}+\left\|a^{\ell}\right\|_{\dot{B}_{2,1}^{\frac{n}{2}}}\right)+\|a\|_{\dot{B}_{p, 1}^{\frac{n}{p}}}\left(\left\|\mathbf{u}^{h}\right\|_{\dot{B}_{p, 1}^{\frac{n}{p}}}+\left\|\mathbf{u}^{\ell}\right\|_{\dot{B}_{2,1}^{\frac{n}{2}}}\right) \\
& \lesssim\left\|\mathbf{u}^{\ell}\right\|_{\dot{B}_{2,1}^{2}}^{2}+\left\|\mathbf{u}^{h}\right\|_{\dot{B}_{p, 1}^{\frac{n}{p}}}^{2}+\left\|a^{\ell}\right\|_{\dot{B}_{2,1}^{\frac{n}{2}}}^{2}+\left\|a^{h}\right\|_{\dot{B}_{p, 1}^{\frac{n}{p}}}^{2} \\
& \lesssim\left(\left\|\left(a^{\ell}, \mathbf{u}^{\ell}\right)\right\|_{\dot{B}_{2,1}^{\frac{n}{2}-1}}+\left\|\mathbf{u}^{h}\right\|_{\dot{B}_{p, 1}^{\frac{n}{p}-1}}\right)\left(\left\|\left(a^{\ell}, \mathbf{u}^{\ell}\right)\right\|_{\dot{B}_{2,1}^{\frac{n}{2}+1}}+\left\|\mathbf{u}^{h}\right\|_{\dot{B}_{p, 1}^{\frac{n}{p}+1}}\right)+\left\|a^{h}\right\|_{\dot{B}_{p, 1}^{\frac{n}{p}}}^{2} \\
\|I(a)(\operatorname{div} \mathbb{T}-\nabla \eta)\|_{\dot{B}_{2,1}^{\frac{n}{2}-1}}^{\ell} & \lesssim\|I(a)\|_{\dot{B}_{p, 1}^{\frac{n}{p}}}\left(\left\|(\operatorname{div} \mathbb{T}-\nabla \eta)^{\ell}\right\|_{\dot{B}_{2,1}^{\frac{n}{2}-1}}+\left\|(\operatorname{div} \mathbb{T}-\nabla \eta)^{h}\right\|_{\dot{B}_{p, 1}^{\frac{n}{p}-1}}\right) \\
& \lesssim\left(\left\|a^{\ell}\right\|_{\dot{B}_{2,1}^{\frac{n}{2}-1}}+\left\|a^{h}\right\|_{\dot{B}_{p, 1}^{\frac{n}{p}}}\right)\left(\left\|\mathbb{T}^{\ell}\right\|_{\dot{B}_{2,1}^{\frac{n}{2}}}+\left\|\mathbb{T}^{h}\right\|_{\dot{B}_{p, 1}^{\frac{n}{p}}}+\left\|\eta^{\ell}\right\|_{\dot{B}_{2,1}^{\frac{n}{2}}}+\left\|\eta^{h}\right\|_{\dot{B}_{p, 1}^{\frac{n}{p}+1}}\right), \\
\|(1-I(a)) \eta \nabla \eta\|_{\dot{B}_{2,1}^{\ell}-1}^{\ell} & \lesssim\left(1+\|I(a)\|_{\left.\dot{B}_{p, 1}^{\frac{n}{p}}\right)}\left(\left\|(\eta \nabla \eta)^{\ell}\right\|_{\dot{B}_{2,1}^{\frac{n}{2}-1}}+\left\|(\eta \nabla \eta)^{h}\right\|_{\left.\dot{B}_{p, 1}^{\frac{n}{p}-1}\right)}\right)\right. \\
& \lesssim\left(\left\|a^{\ell}\right\|_{\dot{B}_{2,1}^{\frac{n}{2}-1}}+\left\|a^{h}\right\|_{\dot{B}_{p, 1}^{\frac{n}{p}}}\right)\left(\left\|\eta^{\ell}\right\|_{\dot{B}_{2,1}^{\frac{n}{2}-2}}\left\|\eta^{\ell}\right\|_{\dot{B}_{2,1}^{\frac{n}{2}}}+\left\|\eta^{h}\right\|_{\dot{B}_{p, 1}^{\frac{n}{p}-1}}\left\|\eta^{h}\right\|_{\dot{B}_{p, 1}^{\frac{n}{p}+1}}\right) .
\end{aligned}
$$

Along the same lines, one can deal with the rest two terms in $G(a, \mathbf{u}, \eta, \mathbb{T})$, thus, substituting the above inequality into (4.15) leads to the lemma, and we complete the proof of Lemma 4.9 . 
4.2. The estimates in the high frequency. In this subsection, we are concerned with the estimates in the high frequency part.

Lemma 4.16. Under the conditions in Theorem 1.3, there holds

$$
\begin{aligned}
& \left\|\left(\mathbf{u}^{h}, \eta^{h}\right)\right\|_{\widetilde{L}_{t}^{\infty}\left(\dot{B}_{p, 1}^{\frac{n}{p}-1}\right)}+\left\|\left(a^{h}, \mathbb{T}^{h}\right)\right\|_{\widetilde{L}_{t}^{\infty}\left(\dot{B}_{p, 1}^{\frac{n}{p}}\right)}+\mu\left\|\mathbf{u}^{h}\right\|_{L_{t}^{1}\left(\dot{B}_{p, 1}^{\frac{n}{p}+1}\right)}+\varepsilon\left\|\eta^{h}\right\|_{L_{t}^{1}\left(\dot{B}_{p, 1}^{\frac{n}{p}+1}\right)} \\
& \quad+\varepsilon\left\|\mathbb{T}^{h}\right\|_{L_{t}^{1}\left(\dot{B}_{p, 1}^{\frac{n}{p}+2}\right)}+\nu^{-1}\left\|a^{h}\right\|_{L_{t}^{1}\left(\dot{B}_{p, 1}^{\frac{n}{p}}\right)}+\frac{A_{0}}{2 \lambda_{1}}\left\|\mathbb{T}^{h}\right\|_{L_{t}^{1}\left(\dot{B}_{p, 1}^{\frac{n}{p}}\right)} \\
& \quad \lesssim\left\|\left(\mathbf{u}_{0}^{h}, \eta_{0}^{h}\right)\right\|_{\dot{B}_{p, 1}^{\frac{n}{p}-1}}+\left\|\left(a_{0}^{h}, \mathbb{T}_{0}^{h}\right)\right\|_{\dot{B}_{p, 1}^{\frac{n}{p}}}+\int_{0}^{t} \mathcal{N}_{1}(s) d s
\end{aligned}
$$

with $\mathcal{N}_{1}(t)$ defined in (4.11).

Proof. By a standard energy estimates, we can get from (4.12) that

$$
\begin{aligned}
& \left\|\mathcal{P} \mathbf{u}^{h}\right\|_{\widetilde{L}_{t}^{\infty}\left(\dot{B}_{p, 1}^{\frac{n}{p}-1}\right)}+\mu\left\|\mathcal{P} \mathbf{u}^{h}\right\|_{L_{t}^{1}\left(\dot{B}_{p, 1}^{\frac{n}{p}+1}\right)} \\
& \quad \lesssim\left\|\mathcal{P} \mathbf{u}_{0}^{h}\right\|_{\dot{B}_{p, 1}^{\frac{n}{p}-1}}+\left\|(\operatorname{div} \mathbb{T})^{h}\right\|_{L_{t}^{1}\left(\dot{B}_{p, 1}^{\frac{n}{p}-1}\right)} \\
& \quad+\left\|(\mathbf{u} \cdot \nabla \mathbf{u})^{h}\right\|_{L_{t}^{1}\left(\dot{B}_{p, 1}^{\frac{n}{p}-1}\right)}+\left\|(G(a, \mathbf{u}, \eta, \mathbb{T}))^{h}\right\|_{L_{t}^{1}\left(\dot{B}_{p, 1}^{\frac{n}{p}-1}\right)}
\end{aligned}
$$

By the product law in Lemma 2.5, we have

$$
\begin{aligned}
\left\|(\mathbf{u} \cdot \nabla \mathbf{u})^{h}\right\|_{\dot{B}_{p, 1}^{\frac{n}{p}-1}} & \lesssim\|\mathbf{u}\|_{\dot{B}_{p, 1}^{\frac{n}{p}-1}}\|\mathbf{u}\|_{\dot{B}_{p, 1}^{\frac{n}{p}+1}} \\
& \lesssim\left(\left\|\mathbf{u}^{\ell}\right\|_{\dot{B}_{2,1}^{\frac{n}{2}-1}}+\left\|\mathbf{u}^{h}\right\|_{\dot{B}_{p, 1}^{\frac{n}{p}-1}}\right)\left(\left\|\mathbf{u}^{\ell}\right\|_{\dot{B}_{2,1}^{\frac{n}{2}+1}}+\left\|\mathbf{u}^{h}\right\|_{\dot{B}_{p, 1}^{\frac{n}{p}+1}}\right) .
\end{aligned}
$$

The first two terms of $G(a, \mathbf{u}, \eta, \mathbb{T})$ can be estimated in the same way as (4.19). To bound the third term of $G(a, \mathbf{u}, \eta, \mathbb{T})$, we use Lemmas $2.5,2.12$ and interpolation inequality to get

$$
\begin{aligned}
& \|I(a)(\operatorname{div} \mathbb{T}-\nabla \eta)\|_{\dot{B}_{p, 1}^{\frac{n}{p}-1}}^{h} \lesssim\|I(a)\|_{\dot{B}_{p, 1}^{\frac{n}{p}}}\|\operatorname{div} \mathbb{T}-\nabla \eta\|_{\dot{B}_{p, 1}^{\frac{n}{p}-1}} \\
& \quad \lesssim\|I(a)\|_{\dot{B}_{p, 1}^{\frac{n}{p}}}\left(\left\|(\operatorname{div} \mathbb{T}-\nabla \eta)^{\ell}\right\|_{\dot{B}_{2,1}^{\frac{n}{2}-1}}+\left\|(\operatorname{div} \mathbb{T}-\nabla \eta)^{h}\right\|_{\dot{B}_{p, 1}^{\frac{n}{p}-1}}\right) \\
& \quad \lesssim\left(\left\|a^{\ell}\right\|_{\dot{B}_{2,1}^{\frac{n}{2}-1}}+\left\|a^{h}\right\|_{\dot{B}_{p, 1}^{\frac{n}{p}}}\right)\left(\left\|\mathbb{T}^{\ell}\right\|_{\dot{B}_{2,1}^{\frac{n}{2}}}+\left\|\mathbb{T}^{h}\right\|_{\dot{B}_{p, 1}^{\frac{n}{p}}}+\left\|\eta^{\ell}\right\|_{\dot{B}_{2,1}^{\frac{n}{2}}}+\left\|\eta^{h}\right\|_{\dot{B}_{p, 1}^{\frac{n}{p}}}\right) \\
& \quad \lesssim\left(\left\|a^{\ell}\right\|_{\dot{B}_{2,1}^{\frac{n}{2}-1}}+\left\|a^{h}\right\|_{\dot{B}_{p, 1}^{\frac{n}{p}}}\right)\left(\left\|\mathbb{T}^{\ell}\right\|_{\dot{B}_{2,1}^{\frac{n}{2}}}+\left\|\mathbb{T}^{h}\right\|_{\dot{B}_{p, 1}^{\frac{n}{p}}}+\left\|\eta^{\ell}\right\|_{\dot{B}_{2,1}^{\frac{n}{2}}}+\left\|\eta^{h}\right\|_{\dot{B}_{p, 1}^{\frac{n}{p}+1}}\right) .
\end{aligned}
$$

Similarly,

$$
\begin{aligned}
\|( & -I(a)) \eta \nabla \eta \|_{\dot{B}_{p, 1}^{\frac{n}{p}-1}}^{h} \\
& \lesssim\left(1+\|I(a)\|_{\dot{B}_{p, 1}^{\frac{n}{p}}}\right)\left(\left\|(\eta \nabla \eta)^{\ell}\right\|_{\dot{B}_{2,1}^{\frac{n}{2}-1}}+\left\|(\eta \nabla \eta)^{h}\right\|_{\dot{B}_{p, 1}^{\frac{n}{p}-1}}\right) \\
& \lesssim\left(\left\|a^{\ell}\right\|_{\dot{B}_{2,1}^{\frac{n}{2}-1}}+\left\|a^{h}\right\|_{\dot{B}_{p, 1}^{p}}\right)\left(\left\|\eta^{\ell}\right\|_{\dot{B}_{2,1}^{\frac{n}{2}}}^{2}+\left\|\eta^{h}\right\|_{\dot{B}_{p, 1}^{\frac{n}{p}}}^{2}\right) \\
& \lesssim\left(\left\|a^{\ell}\right\|_{\dot{B}_{2,1}^{\frac{n}{2}-1}}+\left\|a^{h}\right\|_{\dot{B}_{p, 1}^{\frac{n}{p}}}\right)\left(\left\|\eta^{\ell}\right\|_{\dot{B}_{2,1}^{\frac{n}{2}-2}}\left\|\eta^{\ell}\right\|_{\dot{B}_{2,1}^{\frac{n}{2}}}+\left\|\eta^{h}\right\|_{\dot{B}_{p, 1}^{\frac{n}{p}-1}}\left\|\eta^{h}\right\|_{\dot{B}_{p, 1}^{\frac{n}{p}+1}}\right) .
\end{aligned}
$$

Inserting the above estimates into (4.18), we have

$$
\left\|\mathcal{P} \mathbf{u}^{h}\right\|_{\widetilde{L}_{t}^{\infty}\left(\dot{B}_{p, 1}^{\frac{n}{p}-1}\right)}+\mu\left\|\mathcal{P} \mathbf{u}^{h}\right\|_{L_{t}^{1}\left(\dot{B}_{p, 1}^{\frac{n}{p}+1}\right)} \lesssim\left\|\mathcal{P} \mathbf{u}_{0}^{h}\right\|_{\dot{B}_{p, 1}^{\frac{n}{p}-1}}+\|\mathbb{T}\|_{L_{t}^{1}\left(\dot{B}_{p, 1}^{\frac{n}{p}}\right)}^{h}+\int_{0}^{t} \mathcal{N}_{1}(s) d s
$$

with $\mathcal{N}_{1}(t)$ defined in (4.11). 
Similarly, from the second equation of (1.5), we have

$$
\begin{aligned}
& \left\|\eta^{h}\right\|_{\widetilde{L}_{t}^{\infty}\left(\dot{B}_{p, 1}^{\frac{n}{p}-1}\right)}+\varepsilon\left\|\eta^{h}\right\|_{L_{t}^{1}\left(\dot{B}_{p, 1}^{\frac{n}{p}+1}\right)} \\
& \quad \lesssim\left\|\eta_{0}^{h}\right\|_{\dot{B}_{p, 1}^{\frac{n}{p}-1}}+\int_{0}^{t}\left\|(\eta \operatorname{div} \mathbf{u})^{h}\right\|_{\dot{B}_{p, 1}^{\frac{n}{p}-1}} d s \\
& \quad+\int_{0}^{t}\|\operatorname{div} \mathbf{u}\|_{L^{\infty}}\left\|\eta^{h}\right\|_{\dot{B}_{p, 1}^{\frac{n}{p}-1}} d s+\int_{0}^{t} \sum_{j \geq j_{0}} 2^{\left(\frac{n}{p}-1\right) j}\left\|\left[\dot{\Delta}_{j}, \mathbf{u} \cdot \nabla\right] \eta\right\|_{L^{p}} d s
\end{aligned}
$$

and from the third equation of (1.5), we have

$$
\begin{aligned}
& \left\|\mathbb{T}^{h}\right\|_{\widetilde{L}_{t}^{\infty}\left(\dot{B}_{p, 1}^{p}\right)}+\frac{A_{0}}{2 \lambda_{1}}\left\|\mathbb{T}^{h}\right\|_{L_{t}^{1}\left(\dot{B}_{p, 1}^{\frac{n}{p}}\right)}+\varepsilon\left\|\mathbb{T}^{h}\right\|_{L_{t}^{1}\left(\dot{B}_{p, 1}^{\frac{n}{p}+2}\right)} \\
& \lesssim\left\|\mathbb{T}_{0}^{h}\right\|_{\dot{B}_{p, 1}^{\frac{n}{p}}}+\frac{\kappa A_{0}}{2 \lambda_{1}} \int_{0}^{t}\left\|\eta^{h}\right\|_{\dot{B}_{p, 1}^{\frac{n}{p}}} d s+\int_{0}^{t}\left\|(F(\mathbb{T}, \mathbf{u}))^{h}\right\|_{\dot{B}_{p, 1}^{\frac{n}{p}}} d s \\
& \quad+\int_{0}^{t}\|\operatorname{div} \mathbf{u}\|_{L^{\infty}}\left\|\mathbb{T}^{h}\right\|_{\dot{B}_{p, 1}^{\frac{n}{p}}} d s+\int_{0}^{t} \sum_{j \geq j_{0}} 2^{\frac{n}{p} j}\left\|\left[\dot{\Delta}_{j}, \mathbf{u} \cdot \nabla\right] \mathbb{T}\right\|_{L^{p}} d s .
\end{aligned}
$$

Due to Lemma 2.5 and Lemma 2.11, we get

$$
\begin{aligned}
& \sum_{j \geq j_{0}} 2^{\left(\frac{n}{p}-1\right) j}\left\|\left[\dot{\Delta}_{j}, \mathbf{u} \cdot \nabla\right] \eta\right\|_{L^{p}}+\left\|(\eta \operatorname{div} \mathbf{u})^{h}\right\|_{\dot{B}_{p, 1}^{\frac{n}{p}-1}} \\
& \quad \lesssim\|\eta\|_{\dot{B}_{p, 1}^{\frac{n}{p}-1}}\|\nabla \mathbf{u}\|_{\dot{B}_{p, 1}^{\frac{n}{p}}} \\
& \quad \lesssim\left(\left\|\eta^{\ell}\right\|_{\dot{B}_{2,1}^{\frac{n}{2}-2}}+\left\|\eta^{h}\right\|_{\dot{B}_{p, 1}^{\frac{n}{p}-1}}\right)\left(\left\|\mathbf{u}^{\ell}\right\|_{\dot{B}_{2,1}^{\frac{n}{2}+1}}+\left\|\mathbf{u}^{h}\right\|_{\dot{B}_{p, 1}^{\frac{n}{p}+1}}\right)
\end{aligned}
$$

and

$$
\begin{aligned}
& \sum_{j \geq j_{0}} 2^{\frac{n}{p} j}\left\|\left[\dot{\Delta}_{j}, \mathbf{u} \cdot \nabla\right] \mathbb{T}\right\|_{L^{p}}+\left\|(F(\mathbb{T}, \mathbf{u}))^{h}\right\|_{\dot{B}_{p, 1}^{\frac{n}{p}}} \\
& \quad \lesssim\|\mathbb{T}\|_{\dot{B}_{p, 1}^{\frac{n}{p}}}\|\nabla \mathbf{u}\|_{\dot{B}_{p, 1}^{\frac{n}{p}}} \\
& \quad \lesssim\left(\|\mathbb{T} \ell\|_{\dot{B}_{2,1}^{\frac{n}{2}}}+\left\|\mathbb{T}^{h}\right\|_{\dot{B}_{p, 1}^{\frac{n}{p}}}\right)\left(\left\|\mathbf{u}^{\ell}\right\|_{\dot{B}_{2,1}^{\frac{n}{2}+1}}+\left\|\mathbf{u}^{h}\right\|_{\dot{B}_{p, 1}^{\frac{n}{p}+1}}\right),
\end{aligned}
$$

from which, we get by summing up (4.23) and (4.24) that

$$
\begin{aligned}
& \left\|\eta^{h}\right\|_{\widetilde{L}_{t}^{\infty}\left(\dot{B}_{p, 1}^{\frac{n}{p}-1}\right)}+\left\|\mathbb{T}^{h}\right\|_{\widetilde{L}_{t}^{\infty}\left(\dot{B}_{p, 1}^{\frac{n}{p}}\right)}+\frac{A_{0}}{2 \lambda_{1}}\left\|\mathbb{T}^{h}\right\|_{L_{t}^{1}\left(\dot{B}_{p, 1}^{\frac{n}{p}}\right)}+\frac{\varepsilon}{2}\left\|\eta^{h}\right\|_{L_{t}^{1}\left(\dot{B}_{p, 1}^{\frac{n}{p}+1}\right)}+\varepsilon\left\|\mathbb{T}^{h}\right\|_{L_{t}^{1}\left(\dot{B}_{p, 1}^{\frac{n}{p}+2}\right)} \\
& \quad \lesssim\left\|\eta_{0}^{h}\right\|_{\dot{B}_{p, 1}^{\frac{n}{p}-1}}+\left\|\mathbb{T}_{0}^{h}\right\|_{\dot{B}_{p, 1}^{\frac{n}{p}}} \\
& \quad+\int_{0}^{t}\left(\left\|\eta^{\ell}\right\|_{\dot{B}_{2,1}^{\frac{n}{2}-2}}+\left\|\mathbb{T}^{\ell}\right\|_{\dot{B}_{2,1}^{\frac{n}{2}}}+\left\|\eta^{h}\right\|_{\dot{B}_{p, 1}^{\frac{n}{p}-1}}+\left\|\mathbb{T}^{h}\right\|_{\dot{B}_{p, 1}^{\frac{n}{p}}}\right)\left(\left\|\mathbf{u}^{\ell}\right\|_{\dot{B}_{2,1}^{\frac{n}{2}+1}}+\left\|\mathbf{u}^{h}\right\|_{\dot{B}_{p, 1}^{\frac{n}{p}+1}}\right) d s .
\end{aligned}
$$

To estimate the high frequency part of $(a, \mathcal{Q} \mathbf{u})$, we follow the method used in [13], 24] to introduce a new quantity

$$
\Gamma \stackrel{\text { def }}{=} \mathcal{Q} \mathbf{u}+\nu^{-1}(-\Delta)^{-1} \nabla a
$$

from which and the second equation of (4.13), we have

$$
\begin{aligned}
\partial_{t} \Gamma-\nu \Delta \Gamma= & \nu^{-1} \Gamma-\nu^{-2}(-\Delta)^{-1} \nabla a \\
& +\nu^{-1} \mathcal{Q}(a \mathbf{u})-\mathcal{Q}(\mathbf{u} \cdot \nabla \mathbf{u})+\mathcal{Q} \operatorname{div} \mathbb{T}-\kappa L \nabla \eta+\mathcal{Q} G(a, \mathbf{u}, \eta, \mathbb{T}) .
\end{aligned}
$$


We get by a standard energy argument that

$$
\begin{aligned}
& \left\|\Gamma^{h}\right\|_{\widetilde{L}_{t}^{\infty}\left(\dot{B}_{p, 1}^{\frac{n}{p}-1}\right)}+\nu\left\|\Gamma^{h}\right\|_{L_{t}^{1}\left(\dot{B}_{p, 1}^{\frac{n}{p}+1}\right)} \\
& \quad \lesssim\left\|\Gamma_{0}^{h}\right\|_{\dot{B}_{p, 1}^{\frac{n}{p}-1}}+\int_{0}^{t}\left(\nu^{-1}\left\|\Gamma^{h}\right\|_{\dot{B}_{p, 1}^{\frac{n}{p}-1}}+\nu^{-2}\left\|a^{h}\right\|_{\dot{B}_{p, 1}^{\frac{n}{p}-2}}\right) d s+\int_{0}^{t}\left\|\mathbb{T}^{h}\right\|_{\dot{B}_{p, 1}^{\frac{n}{p}}} d s+\kappa L \int_{0}^{t}\left\|\eta^{h}\right\|_{\dot{B}_{p, 1}^{\frac{n}{p}}} d s \\
& \quad+\int_{0}^{t}\left\|(\mathbf{u} \cdot \nabla \mathbf{u})^{h}\right\|_{\dot{B}_{p, 1}^{\frac{n}{p}-1}} d s+\int_{0}^{t}\left\|(G(a, \mathbf{u}, \eta, \mathbb{T}))^{h}\right\|_{\dot{B}_{p, 1}^{\frac{n}{p}-1}} d s+\nu^{-1} \int_{0}^{t}\left\|(a \mathbf{u})^{h}\right\|_{\dot{B}_{p, 1}^{\frac{n}{p}-1}} d s .
\end{aligned}
$$

Plugging $\mathcal{Q} \mathbf{u}=\Gamma-\nu^{-1}(-\Delta)^{-1} \nabla a$ into the first equation in (4.13) gives

$$
\partial_{t} a+\nu^{-1} a+\mathbf{u} \cdot \nabla a=-\operatorname{div} \Gamma-a \operatorname{div} \mathbf{u},
$$

which further implies that

$$
\begin{aligned}
& \left\|a^{h}\right\|_{\widetilde{L}_{t}^{\infty}\left(\dot{B}_{p, 1}^{\frac{n}{p}}\right)}+\nu^{-1}\left\|a^{h}\right\|_{L_{t}^{1}\left(\dot{B}_{p, 1}^{\frac{n}{p}}\right)} \\
& \lesssim\left\|a_{0}^{h}\right\|_{\dot{B}_{p, 1}^{\frac{n}{p}}}+\int_{0}^{t}\left\|\Gamma^{h}\right\|_{\dot{B}_{p, 1}^{\frac{n}{p}+1}} d s+\int_{0}^{t}\left\|(\operatorname{adiv} \mathbf{u})^{h}\right\|_{\dot{B}_{p, 1}^{\frac{n}{p}+1}} d s \\
& \quad+\int_{0}^{t}\|\operatorname{div} \mathbf{u}\|_{L^{\infty}}\left\|a^{h}\right\|_{\dot{B}_{p, 1}^{\frac{n}{p}}} d s+\int_{0}^{t} \sum_{j \geq j_{0}} 2^{\frac{n}{p} j}\left\|\left[\dot{\Delta}_{j}, \mathbf{u} \cdot \nabla\right] a\right\|_{L^{p}} d s .
\end{aligned}
$$

From Lemma 2.5 and interpolation inequality, we have

$$
\begin{aligned}
\left\|(a \mathbf{u})^{h}\right\|_{\dot{B}_{p, 1}^{\frac{n}{p}-1}} & \lesssim\left\|(a \mathbf{u})^{h}\right\|_{\dot{B}_{p, 1}^{\frac{n}{p}}} \lesssim\|a\|_{\dot{B}_{p, 1}^{\frac{n}{p}}}\|\mathbf{u}\|_{\dot{B}_{p, 1}^{\frac{n}{p}}} \\
& \lesssim\|a\|_{\dot{B}_{p, 1}^{\frac{n}{p}}}^{2}+\|\mathbf{u}\|_{\dot{B}_{p, 1}^{\frac{n}{p}}}^{2} \\
& \lesssim\left\|a^{\ell}\right\|_{\dot{B}_{2,1}^{\frac{n}{2}-1}}\left\|a^{\ell}\right\|_{\dot{B}_{2,1}^{\frac{n}{2}+1}}+\left\|a^{h}\right\|_{\dot{B}_{p, 1}^{\frac{n}{p}}}^{2}+\left\|\mathbf{u}^{\ell}\right\|_{\dot{B}_{2,1}^{\frac{n}{2}-1}}\left\|\mathbf{u}^{\ell}\right\|_{\dot{B}_{2,1}^{\frac{n}{2}+1}}+\left\|\mathbf{u}^{h}\right\|_{\dot{B}_{p, 1}^{\frac{n}{p}-1}}\left\|\mathbf{u}^{h}\right\|_{\dot{B}_{p, 1}^{\frac{n}{p}+1}} .
\end{aligned}
$$

The other nonlinear terms on the right hand side of (4.29), (4.31) can be estimates similarly to (4.19), (4.20), (4.25), (4.26), thus, the combination of (4.29) and (4.31) implies that

$$
\begin{aligned}
& \left\|a^{h}\right\|_{\widetilde{L}_{t}^{\infty}\left(\dot{B}_{p, 1}^{\frac{n}{p}}\right)}+\left\|\Gamma^{h}\right\|_{\widetilde{L}_{t}^{\infty}\left(\dot{B}_{p, 1}^{\frac{n}{p}}-1\right.}+\frac{1}{2 \nu}\left\|a^{h}\right\|_{L_{t}^{1}\left(\dot{B}_{p, 1}^{\frac{n}{p}}\right)}+\frac{\nu}{2}\left\|\Gamma^{h}\right\|_{L_{t}^{1}\left(\dot{B}_{p, 1}^{\frac{n}{p}+1}\right)} \\
& \quad \lesssim\left\|a_{0}^{h}\right\|_{\dot{B}_{p, 1}^{\frac{n}{p}}}+\left\|\Gamma_{0}^{h}\right\|_{\dot{B}_{p, 1}^{\frac{n}{p}-1}}+\left\|\mathbb{T}^{h}\right\|_{L_{t}^{1}\left(\dot{B}_{p, 1}^{\frac{n}{p}}\right)}+\kappa L\left\|\eta^{h}\right\|_{L_{t}^{1}\left(\dot{B}_{p, 1}^{\frac{n}{p}}\right)}+\int_{0}^{t} \mathcal{N}_{1}(s) d s .
\end{aligned}
$$

Combining with (4.22), (4.27) and (4.32) and using $\mathcal{Q} \mathbf{u}=\Gamma-\nu^{-1}(-\Delta)^{-1} \nabla a$, we can obtain (4.17).

4.3. Complete the proof of Theorem 1.3. Now, we can complete the proof of our main Theorem 1.3 by the continuous arguments. To accelerate the proof, we first denote

$$
\begin{gathered}
X(t) \stackrel{\text { def }}{=}\|(a, \mathbf{u})\|_{\widetilde{L}_{t}^{\infty}\left(\dot{B}_{2,1}^{\frac{n}{2}-1}\right)}^{\ell}+\|\eta\|_{\widetilde{L}_{t}^{\infty}\left(\dot{B}_{2,1}^{\frac{n}{2}-2}\right)}^{\ell}+\|\mathbb{T}\|_{\widetilde{L}_{t}^{\infty}\left(\dot{B}_{2,1}^{\frac{n}{2}}\right)}^{\ell}+\|(\mathbf{u}, \eta)\|_{\widetilde{L}_{t}^{\infty}\left(\dot{B}_{p, 1}^{\frac{n}{p}-1}\right)}^{h} \\
+\|(a, \mathbb{T})\|_{\widetilde{L}_{t}^{\infty}\left(\dot{B}_{p, 1}^{\frac{n}{p}}\right)}^{h}+\|(a, \mathbf{u})\|_{L_{t}^{1}\left(\dot{B}_{2,1}^{\frac{n}{2}+1}\right)}^{\ell}+\|(\eta, \mathbb{T})\|_{L_{t}^{1}\left(\dot{B}_{2,1}^{\frac{n}{2}}\right)}^{\ell}+\left\|\mathbb{T}^{\ell}\right\|_{L_{t}^{1}\left(\dot{B}_{2,1}^{\frac{n}{2}+2}\right)} \\
+\|(a, \mathbb{T})\|_{L_{t}^{1}\left(\dot{B_{p}} \frac{n}{p}\right)}^{h}+\|(\mathbf{u}, \eta)\|_{L_{t}^{1}\left(\dot{B}_{p, 1}^{\frac{n}{p}+1}\right)}^{h}+\|\mathbb{T}\|_{L_{t}^{1}\left(\dot{B}_{p, 1}^{\frac{n}{p}+2}\right)}^{h}, \\
X_{0} \stackrel{\text { def }}{=}\left\|\left(a_{0}^{\ell}, \mathbf{u}_{0}^{\ell}\right)\right\|_{\dot{B}_{2,1}^{\frac{n}{2}-1}}+\left\|\eta_{0}^{\ell}\right\|_{\dot{B}_{2,1}^{\frac{n}{2}-2}}+\left\|\mathbb{T}_{0}^{\ell}\right\|_{\dot{B}_{2,1}^{\frac{n}{2}}}+\left\|\left(\mathbf{u}_{0}^{h}, \eta_{0}^{h}\right)\right\|_{\dot{B}_{p, 1}^{\frac{n}{p}-1}}+\left\|\left(a_{0}^{h}, \mathbb{T}_{0}^{h}\right)\right\|_{\dot{B}_{p, 1}^{\frac{n}{p}}} .
\end{gathered}
$$


Multiplying by a suitable large constant on both sides of (4.7) and then pulsing (4.2), we can finally get by combining the resulting inequality with (4.10) that

$$
\begin{aligned}
& \left\|\left(a^{\ell}, \mathbf{u}^{\ell}\right)\right\|_{\widetilde{L}_{t}^{\infty}\left(\dot{B}_{2,1}^{\frac{n}{2}-1}\right)}+\left\|\eta^{\ell}\right\|_{\widetilde{L}_{t}^{\infty}\left(\dot{B}_{2,1}^{\frac{n}{2}-2}\right)}+\left\|\mathbb{T}^{\ell}\right\|_{\widetilde{L}_{t}^{\infty}\left(\dot{B}_{2,1}^{\frac{n}{2}}\right)} \\
& \quad+\mu\left\|\left(a^{\ell}, \mathbf{u}^{\ell}\right)\right\|_{L_{t}^{1}\left(\dot{B}_{2,1}^{\frac{n}{2}+1}\right)}+\frac{\varepsilon}{2}\left\|\eta^{\ell}\right\|_{L_{t}^{1}\left(\dot{B}_{2,1}^{\frac{n}{2}}\right)}+\frac{\varepsilon}{2}\left\|\mathbb{T}^{\ell}\right\|_{L_{t}^{1}\left(\dot{B}_{2,1}^{\frac{n}{2}+2}\right)}+\frac{A_{0}}{4 \lambda_{1}}\left\|\mathbb{T}^{\ell}\right\|_{L_{t}^{1}\left(\dot{B}_{2,1}^{\frac{n}{2}}\right)} \\
& \quad \lesssim\left\|\left(a_{0}^{\ell}, \mathbf{u}_{0}^{\ell}\right)\right\|_{\dot{B}_{2,1}^{\frac{n}{2}-1}}+\left\|\eta_{0}^{\ell}\right\|_{\dot{B}_{2,1}^{\frac{n}{2}-2}}+\left\|\mathbb{T}_{0}^{\ell}\right\|_{\dot{B}_{2,1}^{\frac{n}{2}}}+\int_{0}^{t} \mathcal{N}_{1}(s) d s .
\end{aligned}
$$

Next, combining with (4.17) and (4.33), we can get

$$
X(t) \leq X_{0}+C(X(t))^{2}(1+C X(t)) .
$$

Under the setting of initial data in Theorem 1.3, there exists a positive constant $C_{0}$ such that $X_{0} \leq C_{0} \epsilon$. Due to the local existence result which has been achieved by Theorem 1.1, there exists a positive time $T$ such that

$$
X(t) \leq 2 C_{0} \epsilon, \quad \forall t \in[0, T] .
$$

Let $T^{*}$ be the largest possible time of $T$ for what (4.35) holds. Now, we only need to show $T^{*}=\infty$. By the estimate of (4.34), we can use a standard continuation argument to prove that $T^{*}=\infty$ provided that $\epsilon$ is small enough. We omit the details here. Hence, we finish the proof of Theorem 1.3 .

\section{The Proof of Theorem 1.5}

In this section, we shall follow the method (independent of the spectral analysis) used in [23] and [44 to get the decay rate of the solutions constructed in the previous section. For convenience, we assume all the coefficients appeared in (1.1) equal to one. From the proof of Theorem 1.3, we can get the following inequality (see the derivation of (4.33) and (4.17) for more details):

$$
\begin{aligned}
& \frac{d}{d t}\left(\|(a, \mathbf{u})\|_{\dot{B}_{2,1}^{\frac{n}{2}-1}}^{\ell}+\|\eta\|_{\dot{B}_{2,1}^{\frac{n}{2}-2}}^{\ell}+\|\mathbb{T}\|_{\dot{B}_{2,1}^{\frac{n}{2}}}^{\ell}+\|(\mathbf{u}, \eta)\|_{\dot{B}_{p, 1}^{\frac{n}{p}-1}}^{h}+\|(a, \mathbb{T})\|_{\dot{B}_{p, 1}^{\frac{n}{p}}}^{h}\right) \\
& \quad+\|(a, \mathbf{u})\|_{\dot{B}_{2,1}^{\ell}}^{\ell}+\|(\eta, \mathbb{T})\|_{\dot{B}_{2,1}^{\frac{n}{2}}}^{\ell}+\|a\|_{\dot{B}_{p, 1}^{\frac{n}{p}}}^{h}+\|(\mathbf{u}, \eta)\|_{\dot{B}_{p, 1}^{\frac{n}{p}+1}}^{h}+\| \mathbb{T}_{\dot{B}_{p, 1}^{\frac{n}{p}+2}}^{h} \\
& \quad \lesssim\left(\left\|\left(a^{\ell}, \mathbf{u}^{\ell}\right)\right\|_{\dot{B}_{2,1}^{\frac{n}{2}-1}}+\left\|\mathbf{u}^{h}\right\|_{\dot{B}_{p, 1}^{\frac{n}{p}-1}}\right)\left(\left\|\mathbf{u}^{h}\right\|_{\dot{B}_{p, 1}^{\frac{n}{p}+1}}+\left\|\left(a^{\ell}, \mathbf{u}^{\ell}\right)\right\|_{\dot{B}_{2,1}^{\frac{n}{2}+1}}+\left\|\eta^{\ell}\right\|_{\dot{B}_{2,1}^{\frac{n}{2}}}+\left\|\eta^{h}\right\|_{\dot{B}_{p, 1}^{\frac{n}{p}}}\right) \\
& \quad+\left(\left\|a^{\ell}\right\|_{\dot{B}_{2,1}^{\frac{n}{2}-1}}+\left\|\left(a^{h}, \mathbb{T}^{h}\right)\right\|_{\dot{B}_{p, 1}^{\frac{n}{p}}}\right)\left(\left\|\left(a^{h}, \mathbb{T}^{h}\right)\right\|_{\dot{B}_{p, 1}^{\frac{n}{p}}}+\left\|\eta^{\ell}\right\|_{\dot{B}_{2,1}^{\frac{n}{2}-2}}\left\|\eta^{\ell}\right\|_{\dot{B}_{2,1}^{\frac{n}{2}}}+\left\|\eta^{h}\right\|_{\dot{B}_{p, 1}^{\frac{n}{p}-1}}\left\|\eta^{h}\right\|_{\dot{B}_{p, 1}^{\frac{n}{p}+1}}\right) \\
& \quad+\left(\left\|a^{\ell}\right\|_{\dot{B}_{2,1}^{\frac{n}{2}-1}}+\left\|\mathbb{T}^{\ell}\right\|_{\dot{B}_{2,1}^{\frac{n}{2}}}+\left\|a^{h}\right\|_{\dot{B}_{p, 1}^{\frac{n}{p}}}\right)\left(\left\|\mathbb{T}^{\ell}\right\|_{\dot{B}_{2,1}^{\frac{n}{2}}}+\left\|\mathbb{T}^{h}\right\|_{\dot{B}_{p, 1}^{\frac{n}{p}}}+\left\|\eta^{\ell}\right\|_{\dot{B}_{2,1}^{\frac{n}{2}}}+\left\|\eta^{h}\right\|_{\left.\dot{B}_{p, 1}^{\frac{n}{p}+1}\right) .}\right.
\end{aligned}
$$

By Theorem 1.3, the following estimate holds:

$$
\|(a, \mathbf{u})\|_{\widetilde{L}_{t}^{\infty}\left(\dot{B}_{2,1}^{\frac{n}{2}-1}\right)}^{\ell}+\|\eta\|_{\widetilde{L}_{t}^{\infty}\left(\dot{B}_{2,1}^{\frac{n}{2}-2}\right)}^{\ell}+\|\mathbb{T}\|_{\widetilde{L}_{t}^{\infty}\left(\dot{B}_{2,1}^{\frac{n}{2}}\right)}^{\ell}+\|(\mathbf{u}, \eta)\|_{\widetilde{L}_{t}^{\infty}\left(\dot{B}_{p, 1}^{p}-1\right.}^{h}+\|(a, \mathbb{T})\|_{\widetilde{L}_{t}^{\infty}\left(\dot{B}_{p, 1}^{\frac{n}{p}}\right)}^{h} \leq c_{0},
$$

from which we can infer from (5.1) that

$$
\begin{aligned}
& \frac{d}{d t}\left(\|(a, \mathbf{u})\|_{\dot{B}_{2,1}^{\frac{n}{2}-1}}^{\ell}+\|\eta\|_{\dot{B}_{2,1}^{\frac{n}{2}-2}}^{\ell}+\|\mathbb{T}\|_{\dot{B}_{2,1}^{\frac{n}{2}}}^{\ell}+\|(\mathbf{u}, \eta)\|_{\dot{B}_{p, 1}^{\frac{n}{p}-1}}^{h}+\|(a, \mathbb{T})\|_{\dot{B}_{p, 1}^{\frac{n}{p}}}^{h}\right) \\
& \quad+\bar{c}\left(\|(a, \mathbf{u})\|_{\dot{B}_{2,1}^{\frac{n}{2}+1}}^{\ell}+\|(\eta, \mathbb{T})\|_{\dot{B}_{2,1}^{\frac{n}{2}}}^{\ell}+\|a\|_{\dot{B}_{p, 1}^{\frac{n}{p}}}^{h}+\|(\mathbf{u}, \eta)\|_{\dot{B}_{p, 1}^{\frac{n}{p}+1}}^{h}+\|\mathbb{T}\|_{\dot{B}_{p, 1}^{\frac{n}{p}+2}}^{h}\right) \leq 0 .
\end{aligned}
$$


In order to derive the decay estimate of the solutions given in Theorem 1.3, we need to get a Lyapunov-type differential inequality from (5.3).

According to (5.2) and embedding relation in the high frequency, it's obvious for any $\beta>0$ that

$$
\|\mathbb{T}\|_{\dot{B}_{2,1}^{\frac{n}{2}}}^{h} \geq C\left(\|\mathbb{T}\|_{\dot{B}_{2,1}^{\frac{n}{2}}}^{h}\right)^{1+\beta}
$$

and

$$
\|a\|_{\dot{B}_{p, 1}^{\frac{n}{p}}}^{h}+\|(\mathbf{u}, \eta)\|_{\dot{B}_{p, 1}^{\frac{n}{p}+1}}^{h}+\|\mathbb{T}\|_{\dot{B}_{p, 1}^{\frac{n}{p}+2}}^{h} \geq C\left(\|(\mathbf{u}, \eta)\|_{\dot{B}_{p, 1}^{\frac{n}{p}-1}}^{h}+\|(a, \mathbb{T})\|_{\dot{B}_{p, 1}^{\frac{n}{p}}}^{h}\right)^{1+\beta}
$$

Thus, to get the Lyapunov-type inequality of the solutions, we only need to control the norm of $\|(a, \mathbf{u})\|_{\dot{B}_{2,1}^{2}+1}^{\ell}+\|(\eta, \mathbb{T})\|_{\dot{B}_{2,1}^{\frac{n}{2}}}^{\ell}$. This process can be obtained from the fact that the solutions constructed in Theorem 1.3 can propagate the regularity of the initial data in Besov space with low regularity, see the following Proposition 5.1. This will ensure that one can use interpolation to get the desired Lyapunov-type inequality.

Proposition 5.1. Let $(a, \mathbf{u}, \eta, \mathbb{T})$ be the solutions constructed in Theorem 1.3. For any $\frac{n}{2}-\frac{2 n}{p} \leq$ $\sigma<\frac{n}{2}-1$, and $\left(a_{0}^{\ell}, \mathbf{u}_{0}^{\ell}\right) \in \dot{B}_{2, \infty}^{\sigma}\left(\mathbb{R}^{n}\right), \eta_{0}^{\ell} \in \dot{B}_{2, \infty}^{\sigma-1}\left(\mathbb{R}^{n}\right), \mathbb{T}_{0}^{\ell} \in \dot{B}_{2, \infty}^{\sigma+1}\left(\mathbb{R}^{n}\right)$, then there exists a constant $C_{0}>0$ depends on the norm of the initial data such that for all $t \geq 0$,

$$
\|(a, \mathbf{u})(t, \cdot)\|_{\dot{B}_{2, \infty}^{\sigma}}^{\ell}+\|\eta(t, \cdot)\|_{\dot{B}_{2, \infty}^{\sigma-1}}^{\ell}+\|\mathbb{T}(t, \cdot)\|_{\dot{B}_{2, \infty}^{\sigma+1}}^{\ell} \leq C_{0}
$$

Proof. To simplify the process of proof, we first define the nonlinear terms in (1.5) as

$$
\begin{aligned}
& f_{1} \stackrel{\text { def }}{=}-\operatorname{div}(a \mathbf{u}), \quad f_{2} \stackrel{\text { def }}{=}-\operatorname{div}(\eta \mathbf{u}), \\
& f_{3} \stackrel{\text { def }}{=}-(\mathbf{u} \cdot \nabla) \mathbb{T}+F(\mathbb{T}, \mathbf{u}), \quad f_{4} \stackrel{\text { def }}{=}-(\mathbf{u} \cdot \nabla) \mathbf{u}+G(a, \eta, \mathbf{u}, \mathbb{T}) .
\end{aligned}
$$

From the first and fourth equations of (1.5), we get by a similar derivation of Lemma 5.1 in [44] that

$$
\|(a, \mathbf{u})\|_{\dot{B}_{2, \infty}^{\sigma}}^{\ell} \lesssim\left\|\left(a_{0}, \mathbf{u}_{0}\right)\right\|_{\dot{B}_{2, \infty}^{\sigma}}^{\ell}+\|(\eta, \mathbb{T})\|_{\widetilde{L}_{t}^{1}\left(\dot{B}_{2, \infty}^{\sigma+1}\right)}^{\ell}+\int_{0}^{t}\left\|\left(f_{1}, f_{4}\right)\right\|_{\dot{B}_{2, \infty}^{\sigma}}^{\ell} d s .
$$

From the second and third equations of (1.5), we get by using Lemma 2.13 in the low frequency that

$$
\|\eta\|_{\dot{B}_{2, \infty}^{\sigma-1}}^{\ell}+\|\eta\|_{\widetilde{L}_{t}^{1}\left(\dot{B}_{2, \infty}^{\sigma+1}\right)}^{\ell} \lesssim\left\|\eta_{0}\right\|_{\dot{B}_{2, \infty}^{\sigma-1}}^{\ell}+\int_{0}^{t}\left\|f_{2}\right\|_{\dot{B}_{2, \infty}^{\sigma-1}}^{\ell} d s
$$

and

$$
\|\mathbb{T}\|_{\dot{B}_{2, \infty}^{\sigma+1}}^{\ell}+\|\mathbb{T}\|_{\widetilde{L}_{t}^{1}\left(\dot{B}_{2, \infty}^{\sigma+1}\right)}^{\ell} \lesssim\left\|\mathbb{T}_{0}\right\|_{\dot{B}_{2, \infty}^{\sigma+1}}^{\ell}+\|\eta\|_{\widetilde{L}_{t}^{1}\left(\dot{B}_{2, \infty}^{\sigma+1}\right)}^{\ell}+\int_{0}^{t}\left\|f_{3}\right\|_{\dot{B}_{2, \infty}^{\sigma+1}}^{\ell} d s .
$$

Multiplying by a suitable large constant on both sides of (5.8) and then pulsing (5.9), we can finally get by combining the resulting inequality with (5.7) that

$$
\begin{aligned}
& \|(a, \mathbf{u})\|_{\dot{B}_{2, \infty}^{\sigma}}^{\ell}+\|\eta\|_{\dot{B}_{2, \infty}^{\sigma-1}}^{\ell}+\|\mathbb{T}\|_{\dot{B}_{2, \infty}^{\sigma+1}}^{\ell} \\
& \lesssim\left\|\left(a_{0}, \mathbf{u}_{0}\right)\right\|_{\dot{B}_{2, \infty}^{\sigma}}^{\ell}+\left\|\eta_{0}\right\|_{\dot{B}_{2, \infty}^{\sigma-1}}^{\ell}+\left\|\mathbb{T}_{0}\right\|_{\dot{B}_{2, \infty}^{\sigma+1}}^{\ell} \\
& \quad+\int_{0}^{t}\left\|\left(f_{1}, f_{4}\right)\right\|_{\dot{B}_{2, \infty}^{\sigma}}^{\ell} d s+\int_{0}^{t}\left\|f_{2}\right\|_{\dot{B}_{2, \infty}^{\sigma-1}}^{\ell} d s+\int_{0}^{t}\left\|f_{3}\right\|_{\dot{B}_{2, \infty}^{\sigma+1}}^{\ell} d s .
\end{aligned}
$$

To estimate the nonlinear terms in $f_{1}, f_{2}, f_{3}, f_{4}$, we claim the following important estimates, which we shall postpone its proof in the Appendix. 
Claim : Let $n=2,3$ and $2 \leq p \leq \min (4,2 n /(n-2))$ additionally, $p \neq 4$ if $n=2$, we have

$$
\begin{aligned}
\|f g\|_{\dot{B}_{2, \infty}^{\sigma}} & \lesssim\|f\|_{\dot{B}_{2, \infty}^{\sigma}}\|g\|_{\dot{B}_{p, 1}^{\frac{n}{p}}}, \quad-\frac{n}{p} \leq \sigma<\frac{n}{p} . \\
\left\|f g^{\ell}\right\|_{\dot{B}_{2, \infty}^{\sigma}}^{\ell} & \lesssim\|f\|_{\dot{B}_{p, 1}^{\frac{n}{p}-1}}\left\|g^{\ell}\right\|_{\dot{B}_{2, \infty}^{\sigma+1}}, \quad \frac{n}{2}-\frac{2 n}{p} \leq \sigma<\frac{n}{2}-1 . \\
\left\|f g^{h}\right\|_{\dot{B}_{2, \infty}^{\sigma}}^{\ell} & \lesssim\|f\|_{\dot{B}_{p, 1}^{\frac{n}{p}-1}}\left\|g^{h}\right\|_{\dot{B}_{p, 1}^{\frac{n}{p}}}, \quad \frac{n}{2}-\frac{2 n}{p} \leq \sigma<\frac{n}{2}-1 .
\end{aligned}
$$

To accelerate the proof, we continue to introduce the following notation:

$$
\begin{aligned}
& \mathcal{E}_{\infty}(t) \stackrel{\text { def }}{=}\|(a, \mathbf{u})\|_{\dot{B}_{2,1}^{\frac{n}{2}-1}}^{\ell}+\|\eta\|_{\dot{B}_{2,1}^{\frac{n}{2}-2}}^{\ell}+\|\mathbb{T}\|_{\dot{B}_{2,1}^{\frac{n}{2}}}^{\ell}+\|(\mathbf{u}, \eta)\|_{\dot{B}_{p, 1}^{\frac{n}{p}-1}}^{h}+\left\|\left(a, \mathbb{T}^{\frac{n}{2}}\right)\right\|_{\dot{B}_{p, 1}^{\frac{n}{p}}}^{h}, \\
& \mathcal{E}_{1}(t) \stackrel{\text { def }}{=}\|(a, \mathbf{u})\|_{\dot{B}_{2,1}^{\frac{n}{2}+1}}^{\ell}+\|(\eta, \mathbb{T})\|_{\dot{B}_{2,1}^{\frac{n}{2}}}^{\ell}+\|a\|_{\dot{B}_{p, 1}^{\frac{n}{p}}}^{h}+\|(\mathbf{u}, \eta)\|_{\dot{B}_{p, 1}^{\frac{n}{p}+1}}^{h}+\|\mathbb{T}\|_{\dot{B}_{p, 1}^{\frac{n}{p}+2}}^{h} .
\end{aligned}
$$

From (5.11), one has

$$
\begin{aligned}
& \left\|\mathbf{u} \cdot \nabla a^{\ell}\right\|_{\dot{B}_{2, \infty}^{\sigma}}^{\ell}+\left\|a \operatorname{div} \mathbf{u}^{\ell}\right\|_{\dot{B}_{2, \infty}^{\sigma}}^{\ell} \\
& \quad \lesssim\left\|\mathbf{u}^{\ell}\right\|_{\dot{B}_{2, \infty}^{\sigma}}\left\|\nabla a^{\ell}\right\|_{\dot{B}_{p, 1}^{\frac{n}{p}}}+\|\mathbf{u}\|_{\dot{B}_{p, 1}^{p}}^{h}\left\|\nabla a^{\ell}\right\|_{\dot{B}_{2, \infty}^{\sigma}}+\left\|a^{\ell}\right\|_{\dot{B}_{2, \infty}^{\sigma}}\left\|\operatorname{div} \mathbf{u}^{\ell}\right\|_{\dot{B}_{p, 1}^{\frac{n}{p}}}+\left\|a^{h}\right\|_{\dot{B}_{p, 1}^{\frac{n}{p}}}\left\|\operatorname{div} \mathbf{u}^{\ell}\right\|_{\dot{B}_{2, \infty}^{\sigma}} \\
& \quad \lesssim\left\|a^{\ell}\right\|_{\dot{B}_{2,1}^{\frac{n}{2}+1}}\left\|\mathbf{u}^{\ell}\right\|_{\dot{B}_{2, \infty}^{\sigma}}+\|\mathbf{u}\|_{\dot{B}_{p, 1}^{\frac{n}{p}+1}}^{h}\left\|a^{\ell}\right\|_{\dot{B}_{2, \infty}^{\sigma}}+\|\mathbf{u}\|_{\dot{B}_{2,1}^{\frac{n}{2}+1}}^{\ell}\|a\|_{\dot{B}_{2, \infty}^{\sigma}}^{\ell}+\|a\|_{\dot{B}_{p, 1}^{\frac{n}{p}}}^{h}\|\mathbf{u}\|_{\dot{B}_{2, \infty}^{\sigma}}^{\ell} \\
& \quad \lesssim \mathcal{E}_{1}(t)\left\|\left(a^{\ell}, \mathbf{u}^{\ell}\right)\right\|_{\dot{B}_{2, \infty}^{\sigma}} .
\end{aligned}
$$

Using the second estimate in (5.12), we have

$$
\begin{aligned}
& \left\|\mathbf{u} \cdot \nabla a^{h}\right\|_{\dot{B}_{2, \infty}^{\sigma}}^{\ell}+\left\|a \operatorname{div} \mathbf{u}^{h}\right\|_{\dot{B}_{2, \infty}^{\sigma}}^{\ell} \\
& \lesssim\|\mathbf{u}\|_{\dot{B}_{2, \infty}^{\sigma+1}}^{\ell}\|\nabla a\|_{\dot{B}_{p, 1}^{\frac{n}{p}-1}}^{h}+\|\nabla a\|_{\dot{B}_{p, 1}^{\frac{n}{p}-1}}^{h}\|\mathbf{u}\|_{\dot{B}_{p, 1}^{\frac{n}{p}}}^{h}+\left(\|a\|_{\dot{B}_{p, 1}^{\frac{n}{p}-1}}^{\ell}+\|a\|_{\dot{B}_{p, 1}^{\frac{n}{p}-1}}^{h}\right)\|\operatorname{div} \mathbf{u}\|_{\dot{B}_{p, 1}^{\frac{n}{p}}}^{h} \\
& \lesssim\|a\|_{\dot{B}_{p, 1}^{\frac{n}{p}}}^{h}\|\mathbf{u}\|_{\dot{B}_{2, \infty}^{\sigma}}^{\ell}+\left(\|a\|_{\dot{B}_{2,1}^{\frac{n}{2}-1}}^{\ell}+\|a\|_{\dot{B}_{p, 1}^{\frac{n}{p}}}^{h}\|\mathbf{u}\|_{\dot{B}_{p, 1}^{\frac{n}{p}+1}}^{h} \lesssim \mathcal{E}_{1}(t)\left\|\mathbf{u}^{\ell}\right\|_{\dot{B}_{2, \infty}^{\sigma}}+\mathcal{E}_{\infty}(t) \mathcal{E}_{1}(t)\right.
\end{aligned}
$$

from which and (5.14) gives

$$
\left\|f_{1}\right\|_{\dot{B}_{2, \infty}^{\sigma}}^{\ell} \lesssim \mathcal{E}_{1}(t)\left\|\left(a^{\ell}, \mathbf{u}^{\ell}\right)\right\|_{\dot{B}_{2, \infty}^{\sigma}}+\mathcal{E}_{\infty}(t) \mathcal{E}_{1}(t)
$$

Along the same lines, we have

$$
\begin{aligned}
\left\|f_{2}\right\|_{\dot{B}_{2, \infty}^{\sigma-1}}^{\ell} & \lesssim\|\operatorname{div}(\eta \mathbf{u})\|_{\dot{B}_{2, \infty}^{\sigma-1}}^{\ell} \lesssim\left\|\eta^{\ell} \mathbf{u}^{\ell}\right\|_{\dot{B}_{2, \infty}^{\sigma}}^{\ell}+\left\|\eta^{h} \mathbf{u}^{\ell}\right\|_{\dot{B}_{2, \infty}^{\sigma}}^{\ell}+\left\|\eta^{\ell} \mathbf{u}^{h}\right\|_{\dot{B}_{2, \infty}^{\sigma}}^{\ell}+\left\|\eta^{h} \mathbf{u}^{h}\right\|_{\dot{B}_{2, \infty}^{\sigma}}^{\ell} \\
& \lesssim\left\|\mathbf{u}^{\ell}\right\|_{\dot{B}_{2, \infty}^{\sigma}}\left(\left\|\eta^{\ell}\right\|_{\dot{B}_{2,1}^{\frac{n}{2}}}+\left\|\eta^{h}\right\|_{\dot{B}_{p, 1}^{\frac{n}{p}+1}}\right)+\left\|\eta^{\ell}\right\|_{\dot{B}_{2, \infty}^{\sigma-1}}\left\|\mathbf{u}^{h}\right\|_{\dot{B}_{p, 1}^{\frac{n}{p}+1}}+\left\|\eta^{h}\right\|_{\dot{B}_{p, 1}^{p}-1}\left\|^{\frac{n}{p}}\right\|_{\dot{B}_{p, 1}^{h}}^{\frac{n}{p}+1} \\
& \lesssim \mathcal{E}_{1}(t)\left(\|\mathbf{u}\|_{\dot{B}_{2, \infty}^{\sigma}}^{\ell}+\|\eta\|_{\dot{B}_{2, \infty}^{\sigma-1}}^{\ell}+\mathcal{E}_{\infty}(t) \mathcal{E}_{1}(t) .\right.
\end{aligned}
$$

To bound the terms in $f_{3}$, we use the decomposition $\mathbf{u}=\mathbf{u}^{\ell}+\mathbf{u}^{h}$, and $\mathbb{T}=\mathbb{T}^{\ell}+\mathbb{T}^{h}$ to get

$$
\begin{aligned}
& \left\|\mathbf{u} \cdot \nabla \mathbb{T}^{\ell}\right\|_{\dot{B}_{2, \infty}^{\sigma+1}}^{\ell}+\left\|\mathbb{T} \operatorname{div} \mathbf{u}^{\ell}\right\|_{\dot{B}_{2, \infty}^{\sigma+1}}^{\ell}+\left\|\mathbf{u}^{\ell} \cdot \nabla \mathbb{T}^{h}\right\|_{\dot{B}_{2, \infty}^{\sigma+1}}^{\ell}+\left\|\mathbb{T}^{\ell} \operatorname{div} \mathbf{u}^{h}\right\|_{\dot{B}_{2, \infty}^{\sigma+1}}^{\ell} \\
& \quad \lesssim\left\|\mathbf{u}^{\ell}\right\|_{\dot{B}_{2, \infty}^{\sigma}}\left\|\nabla \mathbb{T}^{\ell}\right\|_{\dot{B}_{p, 1}^{\frac{n}{p}}}+\|\mathbf{u}\|_{\dot{B}_{p, 1}^{\frac{n}{p}}}^{h}\left\|\nabla \mathbb{T}^{\ell}\right\|_{\dot{B}_{2, \infty}^{\sigma}}+\|\mathbb{T}\|_{\dot{B}_{p, 1}^{\frac{n}{p}}}\left\|\operatorname{div} \mathbf{u}^{\ell}\right\|_{\dot{B}_{2, \infty}^{\sigma}} \\
& \quad+\left\|\mathbf{u}^{\ell}\right\|_{\dot{B}_{2, \infty}^{\sigma}}\left\|\nabla \mathbb{T}^{h}\right\|_{\dot{B}_{p, 1}^{\frac{n}{p}}}+\left\|\mathbb{T}^{\ell}\right\|_{\dot{B}_{2, \infty}^{\sigma+1}}\left\|\operatorname{div} \mathbf{u}^{h}\right\|_{\dot{B}_{p, 1}^{\frac{n}{p}-1}} \\
& \quad \lesssim\left(\left\|\mathbb{T}^{\ell}\right\|_{\dot{B}_{2,1}^{\frac{n}{2}}}+\|\mathbb{T}\|_{\dot{B}_{p, 1}^{\frac{n}{p}+2}}^{h}\left\|\mathbf{u}^{\ell}\right\|_{\dot{B}_{2, \infty}^{\sigma}}+\left(\|\mathbf{u}\|_{\dot{B}_{2,1}^{\ell}}^{\ell}+\|\mathbf{u}\|_{\dot{B}_{p, 1}^{\frac{n}{p}+1}}^{h}\right) \| \mathbb{T}_{\dot{B}_{2, \infty}^{\sigma+1}}^{\ell}\right. \\
& \quad \lesssim \mathcal{E}_{1}(t)\left(\left\|\mathbf{u}^{\ell}\right\|_{\dot{B}_{2, \infty}^{\sigma}}+\|\mathbb{T}\|_{\dot{B}_{2, \infty}^{\sigma+1}}^{\ell}\right) .
\end{aligned}
$$


It follows from (5.13) that

$$
\begin{aligned}
\left\|\mathbf{u}^{h} \cdot \nabla \mathbb{T}^{h}\right\|_{\dot{B}_{2, \infty}^{\sigma+1}}^{\ell}+\left\|\mathbb{T}^{h} \operatorname{div} \mathbf{u}^{h}\right\|_{\dot{B}_{2, \infty}^{\sigma+1}}^{\ell} & \lesssim\left\|\mathbf{u}^{h} \cdot \nabla \mathbb{T}^{h}\right\|_{\dot{B}_{2, \infty}^{\sigma}}^{\ell}+\left\|\mathbb{T}^{h} \operatorname{div} \mathbf{u}^{h}\right\|_{\dot{B}_{2, \infty}^{\sigma}}^{\ell} \\
& \lesssim\|\mathbf{u}\|_{\dot{B}_{p, 1}^{\frac{n}{p}-1}}^{h}\|\nabla \mathbb{T}\|_{\dot{B}_{p, 1}^{\frac{n}{p}}}^{h}+\left\|\mathbb{T}_{\dot{B}_{p, 1}^{\frac{n}{p}-1}}^{h}\right\| \operatorname{div} \mathbf{u} \|_{\dot{B}_{p, 1}^{\frac{n}{p}}}^{h} \\
& \lesssim\|\mathbf{u}\|_{\dot{B}_{p, 1}^{\frac{n}{p}-1}}^{h}\|\mathbb{T}\|_{\dot{B}_{p, 1}^{\frac{n}{p}+2}}^{h}+\|\mathbb{T}\|_{\dot{B}_{p, 1}^{\frac{n}{p}}}^{h}\|\mathbf{u}\|_{\dot{B}_{p, 1}^{\frac{n}{p}+1}}^{h} \\
& \lesssim \mathcal{E}_{\infty}(t) \mathcal{E}_{1}(t) .
\end{aligned}
$$

The term of $\|F(\mathbb{T}, \mathbf{u})\|_{\dot{B}_{2, \infty}^{\sigma+1}}^{\ell}$ can be bounded the same as (5.18), (5.19), thus we can get

$$
\left\|f_{3}\right\|_{\dot{B}_{2, \infty}^{\sigma+1}}^{\ell} \lesssim \mathcal{E}_{1}(t)\left(\left\|\mathbf{u}^{\ell}\right\|_{\dot{B}_{2, \infty}^{\sigma}}+\|\mathbb{T}\|_{\dot{B}_{2, \infty}^{\sigma+1}}^{\ell}\right)+\mathcal{E}_{\infty}(t) \mathcal{E}_{1}(t)
$$

Finally, we have to bound the terms in $f_{4}$. Indeed, the terms $\mathbf{u} \cdot \nabla \mathbf{u}, k(a) \nabla a, I(a)(\Delta \mathbf{u}+\nabla \operatorname{div} \mathbf{u})$, $\eta \nabla \eta$ in $f_{4}$ can be dealt with the same as (5.14), (5.15), (5.17), (5.18), here we omit the details. We only present some representative terms in the following. According to the definition of $I(a)$, it's not hard to check that

$$
I(a)=a-a I(a) .
$$

Now, using (5.12), (5.13) and Lemma 2.12, we get

$$
\begin{aligned}
\|a I(a) \operatorname{div} \mathbb{T}\|_{\dot{B}_{2, \infty}^{\sigma}}^{\ell} & \lesssim\left\|a^{\ell} I(a) \operatorname{div} \mathbb{T}\right\|_{\dot{B}_{2, \infty}^{\sigma}}^{\ell}+\left\|a^{h} I(a) \operatorname{div} \mathbb{T}\right\|_{\dot{B}_{2, \infty}^{\sigma}}^{\ell} \\
\lesssim & \|I(a) \operatorname{div} \mathbb{T}\|_{\dot{B}_{p, 1}^{\frac{n}{p}}}^{\ell}\|a\|_{\dot{B}_{2, \infty}^{\sigma}}^{\ell}+\left\|a^{h}\right\|_{\dot{B}_{p, 1}^{\frac{n}{p}}} \| I(a) \operatorname{div} \mathbb{T}_{\dot{B}_{p, 1}^{\frac{n}{p}-1}} \\
& \lesssim\left(\left\|a^{\ell}\right\|_{\dot{B}_{2,1}^{\frac{n}{2}-1}}+\left\|a^{h}\right\|_{\dot{B}_{p, 1}^{\frac{n}{p}}}\right)\left(\left\|\mathbb{T}^{\ell}\right\|_{\dot{B}_{2,1}^{\frac{n}{2}}}+\left\|\mathbb{T}^{h}\right\|_{\dot{B}_{p, 1}^{p}+2}\right)\|a\|_{\dot{B}_{2, \infty}^{\sigma}}^{\ell} \\
& +\left(\left\|a^{\ell}\right\|_{\dot{B}_{2,1}^{\frac{n}{2}-1}}+\left\|a^{h}\right\|_{\dot{B}_{p, 1}^{\frac{n}{p}}}\right)\left(\left\|\mathbb{T}^{\ell}\right\|_{\dot{B}_{2,1}^{\frac{n}{2}}}+\left\|\mathbb{T}^{h}\right\|_{\dot{B}_{p, 1}^{\frac{n}{p}}}\right)\left\|a^{h}\right\|_{\dot{B}_{p, 1}^{\frac{n}{p}}},
\end{aligned}
$$

from which

$$
\|I(a) \operatorname{div} \mathbb{T}\|_{\dot{B}_{2, \infty}^{\sigma}}^{\ell} \lesssim\left(1+\mathcal{E}_{\infty}(t)\right) \mathcal{E}_{1}(t)\|a\|_{\dot{B}_{2, \infty}^{\sigma}}^{\ell}+\left(1+\mathcal{E}_{\infty}(t)\right) \mathcal{E}_{\infty}(t) \mathcal{E}_{1}(t) .
$$

Similarly,

$$
\|I(a) \nabla \eta\|_{\dot{B}_{2, \infty}^{\sigma}}^{\ell} \lesssim\left(1+\mathcal{E}_{\infty}(t)\right) \mathcal{E}_{1}(t)\|a\|_{\dot{B}_{2, \infty}^{\sigma}}^{\ell}+\left(1+\mathcal{E}_{\infty}(t)\right) \mathcal{E}_{\infty}(t) \mathcal{E}_{1}(t) .
$$

Thanks to our claim (5.11)-(5.13) again, we have

$$
\begin{aligned}
\|I(a) \eta \nabla \eta\|_{\dot{B}_{2, \infty}^{\sigma}}^{\ell} & \lesssim\|I(a)\|_{\dot{B}_{p, 1}^{\frac{n}{p}}}\|\eta \nabla \eta\|_{\dot{B}_{2, \infty}^{\sigma}}^{\ell} \\
& \lesssim\left(\left\|a^{\ell}\right\|_{\dot{B}_{2,1}^{\frac{n}{2}-1}}+\left\|a^{h}\right\|_{\dot{B}_{p, 1}^{\frac{n}{p}}}\right)\left(\left\|\eta^{\ell}\right\|_{\dot{B}_{2,1}^{\frac{n}{2}}}\|\eta\|_{\dot{B}_{2, \infty}^{\sigma-1}}^{\ell}+\left\|\eta^{h}\right\|_{\dot{B}_{p, 1}^{\frac{n}{p}-1}}\left\|\eta^{h}\right\|_{\dot{B}_{p, 1}^{\frac{n}{p}+1}}\right) \\
& \lesssim \mathcal{E}_{\infty}(t) \mathcal{E}_{1}(t)\|\eta\|_{\dot{B}_{2, \infty}^{\sigma-1}}^{\ell}+\left(\mathcal{E}_{\infty}(t)\right)^{2} \mathcal{E}_{1}(t) .
\end{aligned}
$$

In all, collecting the above estimates, we obtain that

$$
\left\|f_{4}\right\|_{\dot{B}_{2, \infty}^{\sigma}}^{\ell} \lesssim\left(1+\mathcal{E}_{\infty}(t)\right) \mathcal{E}_{1}(t)\left(\|(a, \mathbf{u})\|_{\dot{B}_{2, \infty}^{\sigma}}^{\ell}+\|\eta\|_{\dot{B}_{2, \infty}^{\sigma-1}}^{\ell}\right)+\left(1+\mathcal{E}_{\infty}(t)\right) \mathcal{E}_{\infty}(t) \mathcal{E}_{1}(t)
$$

Inserting (5.16), (5.19), (5.20), (5.25) into (5.10) gives

$$
\begin{aligned}
& \|(a, \mathbf{u})\|_{\dot{B}_{2, \infty}^{\sigma}}^{\ell}+\|\eta\|_{\dot{B}_{2, \infty}^{\sigma-1}}^{\ell}+\|\mathbb{T}\|_{\dot{B}_{2, \infty}^{\sigma+1}}^{\ell} \\
& \lesssim\left\|\left(a_{0}, \mathbf{u}_{0}\right)\right\|_{\dot{B}_{2, \infty}^{\sigma}}^{\ell}+\left\|\eta_{0}\right\|_{\dot{B}_{2, \infty}^{\sigma-1}}^{\ell}+\left\|\mathbb{T}_{0}\right\|_{\dot{B}_{2, \infty}^{\sigma+1}}^{\ell}+\int_{0}^{t}\left(1+\mathcal{E}_{\infty}(s)\right) \mathcal{E}_{\infty}(s) \mathcal{E}_{1}(s) d s \\
& \quad+\int_{0}^{t}\left(1+\mathcal{E}_{\infty}(s)\right) \mathcal{E}_{1}(s)\left(\|(a, \mathbf{u})\|_{\dot{B}_{2, \infty}^{\sigma}}^{\ell}+\|\eta\|_{\dot{B}_{2, \infty}^{\sigma-1}}^{\ell}+\|\mathbb{T}\|_{\dot{B}_{2, \infty}^{\sigma+1}}^{\ell}\right) d s
\end{aligned}
$$


Thus, one can employ nonlinear generalisations of the Gronwall's inequality to get

$$
\|(a, \mathbf{u})(t, \cdot)\|_{\dot{B}_{2, \infty}^{\sigma}}^{\ell}+\|\eta(t, \cdot)\|_{\dot{B}_{2, \infty}^{\sigma-1}}^{\ell}+\|\mathbb{T}(t, \cdot)\|_{\dot{B}_{2, \infty}^{\sigma+1}}^{\ell} \leq C_{0}
$$

for all $t \geq 0$, where $C_{0}>0$ depends on the norm of the initial data.

Consequently, we complete the proof of Proposition 5.1.

Now, we are begin to get Lyapunov-type inequality from (5.3). For any $\frac{n}{2}-\frac{2 n}{p} \leq \sigma<\frac{n}{2}-1$, it follows from interpolation inequality that

$$
\begin{aligned}
\|(a, \mathbf{u})\|_{\dot{B}_{2,1}^{\frac{n}{2}-1}}^{\ell} & \leq C\left(\|(a, \mathbf{u})\|_{\dot{B}_{2, \infty}^{\sigma}}^{\ell}\right)^{\theta_{1}}\left(\|(a, \mathbf{u})\|_{\dot{B}_{2,1}^{\frac{n}{2}+1}}^{\ell}\right)^{1-\theta_{1}} \\
& \leq C\left(\|(a, \mathbf{u})\|_{\dot{B}_{2, \infty}^{\sigma}}\right)^{\theta_{1}}\left(\|(a, \mathbf{u})\|_{\dot{B}_{2,1}^{\frac{n}{2}+1}}^{\ell}\right)^{1-\theta_{1}}, \quad \theta_{1}=\frac{4}{n-2 \sigma+2} \in(0,1),
\end{aligned}
$$

this together with Proposition 5.1 implies that

$$
\|(a, \mathbf{u})\|_{\dot{B}_{2,1}^{\frac{n}{2}+1}}^{\ell} \geq c_{0}\left(\|(a, \mathbf{u})\|_{\dot{B}_{2,1}^{\frac{n}{2}-1}}^{\ell}\right)^{\frac{1}{1-\theta_{1}}}
$$

For any $\sigma-1<\frac{n}{2}-2$,

$$
\|\eta\|_{\dot{B}_{2,1}^{\frac{n}{2}-2}}^{\ell} \leq C\left(\|\eta\|_{\dot{B}_{2, \infty}^{\sigma-1}}^{\ell}\right)^{\theta_{1}}\left(\|\eta\|_{\dot{B}_{2,1}^{\frac{n}{2}}}^{\ell}\right)^{1-\theta_{1}}, \quad \theta_{1}=\frac{4}{n-2 \sigma+2} \in(0,1)
$$

which combines with (5.27) implies

$$
\|\eta\|_{\dot{B}_{2,1}^{\frac{n}{2}}}^{\ell} \geq c_{0}\left(\|\eta\|_{\dot{B}_{2,1}^{\frac{n}{2}-2}}^{\ell}\right)^{\frac{1}{1-\theta_{1}}}
$$

Similarly, for any $\sigma+1<\frac{n}{2}$, we deduce from interpolation inequality again that

$$
\|\mathbb{T}\|_{\dot{B}_{2,1}^{2}}^{\ell} \leq C\left(\|\mathbb{T}\|_{\dot{B}_{2, \infty}^{\sigma+1}}^{\ell}\right)^{\theta_{1}}\left(\|\mathbb{T}\|_{\dot{B}_{2,1}^{2}+2}^{\ell}\right)^{1-\theta_{1}}
$$

from which and (5.27), we get

$$
\|\mathbb{T}\|_{\dot{B}_{2,1}^{\frac{n}{2}+2}}^{\ell} \geq c_{0}\left(\|\mathbb{T}\|_{\dot{B}_{2,1}^{\frac{n}{2}}}^{\ell}\right)^{\frac{1}{1-\theta_{1}}}, \quad \theta_{1}=\frac{4}{n-2 \sigma+2} \in(0,1) .
$$

Now, taking $\beta=1+\theta_{1}>0$ in (5.4) and (5.5), then combining with (5.28), (5.29), (5.30), we deduce from (5.3) that

$$
\begin{aligned}
& \frac{d}{d t}\left(\|(a, \mathbf{u})\|_{\dot{B}_{2,1}^{\frac{n}{2}-1}}^{\ell}+\|\eta\|_{\dot{B}_{2,1}^{\frac{n}{2}-2}}^{\ell}+\|\mathbb{T}\|_{\dot{B}_{2,1}^{\frac{n}{2}}}^{\ell}+\|(\mathbf{u}, \eta)\|_{\dot{B}_{p, 1}^{\frac{n}{p}-1}}^{h}+\|(a, \mathbb{T})\|_{\dot{B}_{p, 1}^{\frac{n}{p}}}^{h}\right), \\
& \quad+\widetilde{c}_{0}\left(\|(a, \mathbf{u})\|_{\dot{B}_{2,1}^{\frac{n}{2}-1}}^{\ell}+\|\eta\|_{\dot{B}_{2,1}^{\frac{n}{2}-2}}^{\ell}+\|\mathbb{T}\|_{\dot{B}_{2,1}^{\frac{n}{2}}}^{\ell}+\|(\mathbf{u}, \eta)\|_{\dot{B}_{p, 1}^{\frac{n}{p}-1}}^{h}+\|(a, \mathbb{T})\|_{\dot{B}_{p, 1}^{\frac{n}{p}}}^{h}\right)^{1+\frac{4}{n-2 \sigma-2}} \leq 0 .
\end{aligned}
$$

Solving this differential inequality directly, we obtain

$$
\|(a, \mathbf{u})\|_{\dot{B}_{2,1}^{\frac{n}{2}-1}}^{\ell}+\|\eta\|_{\dot{B}_{2,1}^{\frac{n}{2}-2}}^{\ell}+\|\mathbb{T}\|_{\dot{B}_{2,1}^{\frac{n}{2}}}^{\ell}+\|(\mathbf{u}, \eta)\|_{\dot{B}_{p, 1}^{\frac{n}{p}-1}}^{h}+\|(a, \mathbb{T})\|_{\dot{B}_{p, 1}^{\frac{n}{p}}}^{h} \leq C(1+t)^{-\frac{n-2 \sigma-2}{4}} .
$$

For any $\frac{n}{p}-\frac{n}{2}+\sigma<\gamma_{1}<\frac{n}{p}-1$, by the interpolation inequality we have

$$
\begin{aligned}
\|(a, \mathbf{u})\|_{\dot{B}_{p, 1}^{\gamma_{1}}}^{\ell} & \leq C\|(a, \mathbf{u})\|_{\dot{B}_{2,1}^{\gamma_{1}+\frac{n}{2}-\frac{n}{p}}}^{\ell} \\
& \leq C\left(\|(a, \mathbf{u})\|_{\dot{B}_{2, \infty}^{\sigma}}^{\ell}\right)^{\theta_{2}}\left(\|(a, \mathbf{u})\|_{\dot{B}_{2,1}^{\frac{n}{2}-1}}^{\ell}\right)^{1-\theta_{2}}, \quad \theta_{2}=\frac{\frac{n}{p}-1-\gamma_{1}}{\frac{n}{2}-1-\sigma} \in(0,1),
\end{aligned}
$$

which combines with Proposition 5.1 gives

$$
\|(a, \mathbf{u})\|_{\dot{B}_{p, 1}^{\gamma_{1}}}^{\ell} \leq C(1+t)^{-\frac{\left(\frac{n}{2}-\sigma-1\right)\left(1-\theta_{2}\right)}{2}}=C(1+t)^{-\frac{n}{2}\left(\frac{1}{2}-\frac{1}{p}\right)-\frac{\gamma_{1}-\sigma}{2}}
$$


In the light of $\frac{n}{p}-\frac{n}{2}+\sigma<\gamma_{1}<\frac{n}{p}-1$, we see that

$$
\left\|\left(a^{h}, \mathbf{u}^{h}\right)\right\|_{\dot{B}_{p, 1}^{\gamma_{1}}} \leq C\left(\|a\|_{\dot{B}_{p, 1}^{\frac{n}{p}}}^{h}+\|\mathbf{u}\|_{\dot{B}_{p, 1}^{\frac{n}{p}-1}}^{h}\right) \leq C(1+t)^{-\frac{n-2 \sigma-2}{4}},
$$

from which and (5.33) gives

$$
\begin{aligned}
\|(a, \mathbf{u})\|_{\dot{B}_{p, 1}^{\gamma_{1}}} & \leq C\left(\|(a, \mathbf{u})\|_{\dot{B}_{p, 1}^{\gamma_{1}}}^{\ell}+\|(a, \mathbf{u})\|_{\dot{B}_{p, 1}^{\gamma_{1}}}^{h}\right) \\
& \leq C(1+t)^{-\frac{n}{2}\left(\frac{1}{2}-\frac{1}{p}\right)-\frac{\gamma_{1}-\sigma}{2}}+C(1+t)^{-\frac{d-2 \sigma-2}{4}} \\
& \leq C(1+t)^{-\frac{n}{2}\left(\frac{1}{2}-\frac{1}{p}\right)-\frac{\gamma_{1}-\sigma}{2}} .
\end{aligned}
$$

Hence, thanks to the embedding relation $\dot{B}_{p, 1}^{0}\left(\mathbb{R}^{n}\right) \hookrightarrow L^{p}\left(\mathbb{R}^{n}\right)$, one infer that

$$
\left\|\Lambda^{\gamma_{1}}(a, \mathbf{u})\right\|_{L^{p}} \leq C(1+t)^{-\frac{n}{2}\left(\frac{1}{2}-\frac{1}{p}\right)-\frac{\gamma_{1}-\sigma}{2}} .
$$

For any $\frac{n}{p}-\frac{n}{2}-1+\sigma<\gamma_{2}<\frac{n}{p}-1$, we have

$$
\|\eta\|_{\dot{B}_{p, 1}^{\gamma_{2}}}^{\ell} \leq C\|\eta\|_{\dot{B}_{2,1}^{\gamma_{2}+\frac{n}{2}-\frac{n}{p}}}^{\ell} \leq C\left(\|\eta\|_{\dot{B}_{2, \infty}^{\sigma-1}}^{\ell}\right)^{\theta_{3}}\left(\|\eta\|_{\dot{B}_{2,1}^{\frac{n}{2}-2}}^{\ell}\right)^{1-\theta_{3}}, \quad \theta_{3}=\frac{\frac{n}{p}-\gamma_{2}-2}{\frac{n}{2}-1-\sigma} \in(0,1),
$$

which gives

$$
\|\eta\|_{\dot{B}_{p, 1}^{\gamma_{2}}}^{\ell} \leq C(1+t)^{-\frac{n}{2}\left(\frac{1}{2}-\frac{1}{p}\right)-\frac{\gamma_{2}-\sigma+1}{2}} .
$$

As $\gamma_{2}<\frac{n}{p}-1$, we see in the high frequency part that

$$
\|\eta\|_{\dot{B}_{p, 1}^{\gamma_{2}}}^{h} \leq C\|\eta\|_{\dot{B}_{p, 1}^{\frac{n}{p}-1}}^{h} \leq C(1+t)^{-\frac{n-2 \sigma-2}{4}} .
$$

From (5.34) and (5.35), we have

$$
\|\eta\|_{\dot{B}_{p, 1}^{\gamma_{2}}} \leq C\left(\|\eta\|_{\dot{B}_{p, 1}^{\gamma_{2}}}^{\ell}+\|\eta\|_{\dot{B}_{p, 1}^{\gamma_{2}}}^{h}\right) \leq C(1+t)^{-\frac{n}{2}\left(\frac{1}{2}-\frac{1}{p}\right)-\frac{\gamma_{2}-\sigma+1}{2}}
$$

which further implies for any $\frac{n}{p}-\frac{n}{2}-1+\sigma<\gamma_{2}<\frac{n}{p}-1$, that

$$
\left\|\Lambda^{\gamma_{2}} \eta\right\|_{L^{p}} \leq C(1+t)^{-\frac{n}{2}\left(\frac{1}{2}-\frac{1}{p}\right)-\frac{\gamma_{2}-\sigma+1}{2}} .
$$

For any $\frac{n}{p}-\frac{n}{2}+1+\sigma<\gamma_{3}<\frac{n}{p}$, by the interpolation inequality we have

$$
\|\mathbb{T}\|_{\dot{B}_{p, 1}^{\gamma_{3}}}^{\ell} \leq C\|\mathbb{T}\|_{\dot{B}_{2,1}^{\gamma_{3}+\frac{n}{2}-\frac{n}{p}}}^{\ell} \leq C\left(\|\mathbb{T}\|_{\dot{B}_{2, \infty}^{\sigma+1}}^{\ell}\right)^{\theta_{4}}\left(\|\mathbb{T}\|_{\dot{B}_{2,1}^{\frac{n}{2}}}^{\ell}\right)^{1-\theta_{4}}, \quad \theta_{4}=\frac{\frac{n}{p}-\gamma_{3}}{\frac{n}{2}-1-\sigma} \in(0,1),
$$

which gives

$$
\|\mathbb{T}\|_{\dot{B}_{p, 1}^{\gamma_{3}}}^{\ell} \leq C(1+t)^{-\frac{\left(\frac{n}{2}-\sigma-1\right)\left(1-\theta_{4}\right)}{2}}=C(1+t)^{-\frac{n}{2}\left(\frac{1}{2}-\frac{1}{p}\right)-\frac{\gamma_{3}-\sigma-1}{2}} .
$$

In the light of $\frac{n}{p}-\frac{n}{2}+1+\sigma<\gamma_{3}<\frac{n}{p}$, we see that

$$
\|\mathbb{T}\|_{\dot{B}_{p, 1}^{\gamma_{3}}}^{h} \leq C\|\mathbb{T}\|_{\dot{B}_{p, 1}^{p}}^{h} \leq C(1+t)^{-\frac{n-2 \sigma-2}{4}} .
$$

From (5.36) and (5.37), we have

$$
\|\mathbb{T}\|_{\dot{B}_{p, 1}^{\gamma_{3}}} \leq C\left(\|\mathbb{T}\|_{\dot{B}_{p, 1}^{\gamma_{3}}}^{\ell}+\|\mathbb{T}\|_{\dot{B}_{p, 1}^{\gamma_{3}}}^{h}\right) \leq C(1+t)^{-\frac{n}{2}\left(\frac{1}{2}-\frac{1}{p}\right)-\frac{\gamma_{3}-\sigma-1}{2}},
$$

which further implies for any $\frac{n}{p}-\frac{n}{2}+1+\sigma<\gamma_{3}<\frac{n}{p}$, that

$$
\left\|\Lambda^{\gamma_{3}} \mathbb{T}\right\|_{L^{p}} \leq C(1+t)^{-\frac{n}{2}\left(\frac{1}{2}-\frac{1}{p}\right)-\frac{\gamma_{3}-\sigma-1}{2}} .
$$

This complete the proof of the Theorem 1.5. 


\section{Acknowledgments}

This work is supported by National Natural Science Foundation of China under grant numbers 12001377,11601533, 11971356, National Natural Science Foundation key project of China under grant number 11831003, and Natural Science Foundation of Guangdong Province of China under grant numbers 2018A030313024, 2020B1515310008, and Project of Educational Commission of Guangdong Province of China under grant number 2019KZDZX1007.

Appendix: The PRoOf of our Claim (5.11)-(5.13)

Proof. We first use Bony's decomposition to rewrite

$$
f g=\dot{T}_{f} g+\dot{T}_{g} f+\dot{R}(f, g) .
$$

Due to Hölder's inequality, Bernstein's inequality and the embedding $\dot{B}_{p, 1}^{\frac{n}{p}}\left(\mathbb{R}^{n}\right) \hookrightarrow L^{\infty}\left(\mathbb{R}^{n}\right)$, we can get

$$
\begin{aligned}
\left\|\dot{\Delta}_{j}\left(\dot{T}_{f} g\right)\right\|_{L^{2}} & \lesssim \sum_{|k-j| \leq 4}\left\|\dot{\Delta}_{j}\left(\dot{S}_{k-1} f \dot{\Delta}_{k} g\right)\right\|_{L^{2}} \\
& \lesssim \sum_{|k-j| \leq 4}\left\|\dot{S}_{k-1} f\right\|_{L^{\infty}}\left\|\dot{\Delta}_{k} g\right\|_{L^{2}} \\
& \lesssim \sum_{|k-j| \leq 4}\|f\|_{L^{\infty}}\left\|\dot{\Delta}_{k} g\right\|_{L^{2}} \\
& \lesssim 2^{-\sigma j}\|f\|_{\dot{B}_{p, 1}^{\frac{n}{p}}}\|g\|_{\dot{B}_{2, \infty}^{\sigma}}
\end{aligned}
$$

Similarly, for any $\sigma<\frac{n}{p}$, the second term in (A.1) can be estimated as follows

$$
\begin{aligned}
\left\|\dot{\Delta}_{j}\left(\dot{T}_{g} f\right)\right\|_{L^{2}} & \lesssim \sum_{|k-j| \leq 4} \sum_{k^{\prime} \leq k-2}\left\|\dot{\Delta}_{k^{\prime}} g\right\|_{L^{2 p /(p-2)}}\left\|\dot{\Delta}_{k} f\right\|_{L^{p}} \\
& \lesssim \sum_{|k-j| \leq 4}\left(\sum_{k^{\prime} \leq k-2} 2^{k^{\prime}\left(\frac{n}{p}-\sigma\right)} 2^{k^{\prime} \sigma}\left\|\dot{\Delta}_{k^{\prime}} g\right\|_{L^{2}}\right)\left\|\dot{\Delta}_{k} f\right\|_{L^{p}} \\
& \lesssim 2^{-\sigma j}\|f\|_{\dot{B}_{p, 1}^{\frac{n}{p}}\|g\|_{\dot{B}_{2, \infty}^{\sigma}}}
\end{aligned}
$$

In view of the fact that $\sigma \geq-\frac{n}{p}$, we can deal with the last term in (A.1)

$$
\begin{aligned}
\left\|\dot{\Delta}_{j} \dot{R}(f, g)\right\|_{L^{2}} & \lesssim \sum_{k \geq j-3} \sum_{\left|k-k^{\prime}\right| \leq 1}\left\|\dot{\Delta}_{j}\left(\dot{\Delta}_{k} f \dot{\Delta}_{k^{\prime}} g\right)\right\|_{L^{2}} \\
& \lesssim 2^{\frac{n}{p} j} \sum_{k \geq j-3} \sum_{\left|k-k^{\prime}\right| \leq 1}\left\|\dot{\Delta}_{k} f \dot{\Delta}_{k^{\prime}} g\right\|_{L^{\frac{2 p}{p+2}}} \\
& \lesssim 2^{\frac{n}{p} j} \sum_{k \geq j-3} \sum_{\left|k-k^{\prime}\right| \leq 1} 2^{-k \frac{n}{p}} 2^{k \frac{n}{p}}\left\|\dot{\Delta}_{k} f\right\|_{L^{p}} 2^{-k^{\prime} \sigma} 2^{k^{\prime} \sigma}\left\|\dot{\Delta}_{k^{\prime}} g\right\|_{L^{2}} \\
& \lesssim 2^{\frac{n}{p} j} \sum_{k \geq j-3} 2^{-k\left(\sigma+\frac{n}{p}\right)} d_{k}\|f\|_{\dot{B}_{p, 1}^{\frac{n}{p}}\|g\|_{\dot{B}_{2, \infty}^{\sigma}}} \\
& \lesssim 2^{-\sigma j}\|f\|_{\dot{B}_{p, 1}^{\frac{n}{p}}}\|g\|_{\dot{B}_{2, \infty}^{\sigma}} .
\end{aligned}
$$

The combination of (A.2)-(A.4) gives (5.11). 
Now, we are in a position to prove (5.12). We also use Bony's decomposition to write $f g^{\ell}=\dot{T}_{f} g^{\ell}+\dot{T}_{g^{\ell}} f+\dot{R}\left(f, g^{\ell}\right)$. It follows from the Hölder inequality and the Bernstein inequality to get

$$
\begin{aligned}
\left\|\dot{\Delta}_{j}\left(\dot{T}_{f} g^{\ell}\right)\right\|_{L^{2}} & \lesssim \sum_{|k-j| \leq 4} \sum_{k^{\prime} \leq k-2}\left\|\dot{\Delta}_{k^{\prime}} f\right\|_{L^{\infty}}\left\|\dot{\Delta}_{k} g^{\ell}\right\|_{L^{2}} \\
& \lesssim \sum_{|k-j| \leq 4} \sum_{k^{\prime} \leq k-2} 2^{\frac{n k^{\prime}}{p}}\left\|\dot{\Delta}_{k^{\prime}} f\right\|_{L^{p}}\left\|\dot{\Delta}_{k} g^{\ell}\right\|_{L^{2}} \\
& \lesssim \sum_{|k-j| \leq 4}\left(\sum_{k^{\prime} \leq k-2} 2^{k^{\prime}} 2^{k^{\prime}\left(\frac{n}{p}-1\right)}\left\|\dot{\Delta}_{k^{\prime}} f\right\|_{L^{p}}\right) 2^{-k(\sigma+1)} 2^{k(\sigma+1)}\left\|\dot{\Delta}_{k} g^{\ell}\right\|_{L^{2}} \\
& \lesssim 2^{-\sigma j}\|f\|_{\dot{B}_{p, 1}^{\frac{n}{p}-1}\left\|g^{\ell}\right\|_{\dot{B}_{2, \infty}^{\sigma+1}}}
\end{aligned}
$$

Along the same lines, for any $\sigma<\frac{n}{2}-1$, one has

$$
\begin{aligned}
\left\|\dot{\Delta}_{j}\left(\dot{T}_{g^{\ell}} f\right)\right\|_{L^{2}} & \leq \sum_{|k-j| \leq 4} \sum_{k^{\prime} \leq k-2}\left\|\dot{\Delta}_{j}\left(\dot{\Delta}_{k^{\prime}} g^{\ell} \dot{\Delta}_{k} f\right)\right\|_{L^{2}} \\
& \leq \sum_{|k-j| \leq 4} \sum_{k^{\prime} \leq k-2}\left\|\dot{\Delta}_{k^{\prime}} g^{\ell}\right\|_{L^{\frac{2 p}{p-2}}}\left\|\dot{\Delta}_{k} f\right\|_{L^{p}} \\
& \lesssim \sum_{|k-j| \leq 4} \sum_{k^{\prime} \leq k-2} 2^{k^{\prime}\left(\frac{2 n}{p}-\frac{n}{2}\right)}\left\|\dot{\Delta}_{k^{\prime}} g^{\ell}\right\|_{L^{p}}\left\|\dot{\Delta}_{k} f\right\|_{L^{p}} \\
& \lesssim \sum_{|k-j| \leq 4}\left(\sum_{k^{\prime} \leq k-2} 2^{-k^{\prime}\left(\sigma-\frac{n}{2}+1\right)} 2^{k^{\prime}\left(\sigma+\frac{2 n}{p}-n+1\right)}\left\|\dot{\Delta}_{k^{\prime}} g^{\ell}\right\|_{L^{p}}\right)\left\|\dot{\Delta}_{k} f\right\|_{L^{p}} \\
& \lesssim 2^{-\left(\sigma+\frac{n}{p}-\frac{n}{2}\right) j}\|f\|_{\dot{B}_{p, 1}^{\frac{n}{p}-1}\left\|g^{\ell}\right\|_{\dot{B}_{p, \infty}^{\sigma+\frac{2 n}{p}-n+1}}} \\
& \lesssim 2^{-\left(\sigma+\frac{n}{p}-\frac{n}{2}\right) j}\|f\|_{\dot{B}_{p, 1}^{\frac{n}{p}-1}\left\|g^{\ell}\right\|_{\dot{B}_{2, \infty}^{\sigma+1}}}
\end{aligned}
$$

As $\sigma+\frac{2 n}{p}-\frac{n}{2}>0$, we can deal with the the remainder term as follows:

$$
\begin{aligned}
\left\|\dot{\Delta}_{j} \dot{R}\left(f, g^{\ell}\right)\right\|_{L^{2}} & \lesssim \sum_{k \geq j-3} \sum_{\left|k-k^{\prime}\right| \leq 1}\left\|\dot{\Delta}_{j}\left(\dot{\Delta}_{k} f \dot{\Delta}_{k^{\prime}} g^{\ell}\right)\right\|_{L^{2}} \\
& \lesssim 2^{j\left(\frac{2 n}{p}-\frac{n}{2}\right)} \sum_{k \geq j-3} \sum_{\left|k-k^{\prime}\right| \leq 1}\left\|\dot{\Delta}_{k} f\right\|_{L^{p}} 2^{\left(\frac{n}{2}-\frac{n}{p} k^{\prime}\right)}\left\|\dot{\Delta}_{k^{\prime}} g^{\ell}\right\|_{L^{2}} \\
& \lesssim 2^{j\left(\frac{2 n}{p}-\frac{n}{2}\right)} \sum_{k \geq j-3} 2^{-k\left(\sigma+\frac{2 n}{p}-\frac{n}{2}\right)} d_{k}\|f\|_{\dot{B}_{p, 1}^{\frac{n}{p}-1}}\left\|g^{\ell}\right\|_{\dot{B}_{2, \infty}^{\sigma+1}} \\
& \lesssim 2^{-\sigma j}\|f\|_{\dot{B}_{p, 1}^{\frac{n}{p}-1}\left\|g^{\ell}\right\|_{\dot{B}_{2, \infty}^{\sigma+1}}}
\end{aligned}
$$

Summing up (A.5)-(A.7), noticing $\sigma+\frac{n}{p}-\frac{n}{2} \leq \sigma$, we can obtain (15.12).

Finally, we are concerned with the proof of (5.13). Thanks to Bony's decomposition again

$$
f g^{h}=\dot{T}_{f} g^{h}+\dot{T}_{g^{h}} f+\dot{R}\left(f, g^{h}\right) .
$$

By virtue of the Hölder inequality, Bernstein's inequality and the fact

$$
\frac{n}{2}-1 \leq \frac{n}{p}, \quad \text { and } \quad \sigma<\frac{n}{2}-1
$$


one deduces that

$$
\begin{aligned}
\left\|\dot{\Delta}_{j}\left(\dot{T}_{f} g^{h}\right)\right\|_{L^{2}} & \lesssim \sum_{|k-j| \leq 4}\left(\sum_{k^{\prime} \leq k-2}\left\|\dot{\Delta}_{k^{\prime}} f\right\|_{L^{\frac{2 p}{p-2}}}\right)\left\|\dot{\Delta}_{k} g^{h}\right\|_{L^{p}} \\
& \lesssim \sum_{|k-j| \leq 4}\left(\sum_{k^{\prime} \leq k-2} 2^{k^{\prime}\left(\frac{2 n}{p}-\frac{n}{2}\right)}\left\|\dot{\Delta}_{k^{\prime}} f\right\|_{L^{p}}\right)\left\|\dot{\Delta}_{k} g^{h}\right\|_{L^{p}} \\
& \lesssim \sum_{|k-j| \leq 4}\left(\sum_{k^{\prime} \leq k-2} 2^{k^{\prime}\left(\frac{n}{p}-\frac{n}{2}+1\right)} 2^{k^{\prime}\left(\frac{n}{p}-1\right)}\left\|\dot{\Delta}_{k^{\prime}} f\right\|_{L^{p}}\right) 2^{-k \sigma} 2^{k\left(\sigma+\frac{n}{p}-\frac{n}{2}+1\right)}\left\|\dot{\Delta}_{k} g^{h}\right\|_{L^{p}} \\
& \lesssim 2^{-\sigma j}\|f\|_{\dot{B}_{p, 1}^{\frac{n}{p}-1}\left\|g^{h}\right\|_{\dot{B}_{p, \infty}^{\sigma+\frac{n}{p}-\frac{n}{2}+1}}} \\
& \lesssim 2^{-\sigma j}\|f\|_{\dot{B}_{p, 1}^{\frac{n}{p}-1}}\left\|g^{h}\right\|_{\dot{B}_{p, 1}^{\frac{n}{p}} .}
\end{aligned}
$$

The term $\left\|\dot{\Delta}_{j} \dot{R}\left(f, g^{h}\right)\right\|_{L^{2}}$ can be dealt in a similar manner,

$$
\left\|\dot{\Delta}_{j} \dot{R}\left(f, g^{h}\right)\right\|_{L^{2}} \lesssim 2^{-\sigma j}\|f\|_{\dot{B}_{p, 1}^{\frac{n}{p}-1}}\left\|g^{h}\right\|_{\dot{B}_{p, 1}^{\frac{n}{p}}}
$$

Thanks to Bernstein's inequality, we have

$$
\begin{aligned}
\left\|\dot{\Delta}_{j}\left(\dot{T}_{g^{h}} f\right)\right\|_{L^{2}} & \lesssim \sum_{|k-j| \leq 4} \sum_{k^{\prime} \leq k-2}\left\|\dot{\Delta}_{j}\left(\dot{\Delta}_{k^{\prime}} g^{h} \dot{\Delta}_{k} f\right)\right\|_{L^{2}} \lesssim \sum_{|k-j| \leq 4}\left(\sum_{k^{\prime} \leq k-2}\left\|\dot{\Delta}_{k^{\prime}} g^{h}\right\|_{L^{\frac{2 p}{p-2}}}\right)\left\|\dot{\Delta}_{k} f\right\|_{L^{p}} \\
& \lesssim \sum_{|k-j| \leq 4}\left(\sum_{k^{\prime} \leq k-2} 2^{k^{\prime}\left(\frac{2 n}{p}-\frac{n}{2}\right)}\left\|\dot{\Delta}_{k^{\prime}} g^{h}\right\|_{L^{p}}\right)\left\|\dot{\Delta}_{k} f\right\|_{L^{p}} \\
& \lesssim \sum_{|k-j| \leq 4}\left(\sum_{k^{\prime} \leq k-2} 2^{-k^{\prime}\left(\sigma+1-\frac{n}{2}\right)} 2^{k^{\prime}\left(\sigma+\frac{2 n}{p}-n+1\right)}\left\|\dot{\Delta}_{k^{\prime}} g^{h}\right\|_{L^{p}}\right)\left\|\dot{\Delta}_{k} f\right\|_{L^{p}} \\
& \lesssim 2^{-\left(\sigma-\frac{n}{2}+\frac{n}{p}\right) j}\|f\|_{\dot{B}_{p, 1}^{\frac{n}{p}-1}\left\|g^{h}\right\|_{\dot{B}_{p, \infty}^{\sigma+\frac{2 n}{p}-n+1}}}
\end{aligned}
$$

from which and relations

$$
\frac{n}{p}-\frac{n}{2} \leq 0, \quad \sigma+\frac{2 n}{p}-n+1 \leq \frac{n}{p}
$$

we have

$$
\left\|\dot{T}_{g^{h}} f\right\|_{\dot{B}_{2,1}^{\sigma}}^{\ell} \lesssim\left\|\dot{T}_{g^{h}} f\right\|_{\dot{B}_{2,1}^{\sigma-\frac{n}{2}+\frac{n}{p}}}^{\ell} \lesssim\|f\|_{\dot{B}_{p, 1}^{\frac{n}{p}-1}}\left\|g^{h}\right\|_{\dot{B}_{p, \infty}^{\sigma+\frac{2 n}{p}-n+1}} \lesssim\|f\|_{\dot{B}_{p, 1}^{\frac{n}{p}-1}}\left\|g^{h}\right\|_{\dot{B}_{p, 1}^{p}}
$$

Collecting (A.9), (A.10) and(A.12), the desired estimate (5.13) is obtained.

Consequently, we conclude the proof of (5.11)-(5.13).

\section{REFERENCES}

[1] H. Bahouri, J.Y. Chemin, R. Danchin, Fourier Analysis and Nonlinear Partial Differential Equations. Grundlehren Math. Wiss., vol. 343, Springer-Verlag, Berlin, Heidelberg, 2011.

[2] J.W. Barrett, S. Boyaval, Existence and approximation of a (regularized) Oldroyd-B model, Math. Models Methods Appl. Sci., 21, 1783-1837, 2011.

[3] J.W. Barrett, Y. Lu, E. Süli, Existence of large-data finite-energy global weak solutions to a compressible Oldroyd-B model, Commun. Math. Sci., 15, 1265-1323, 2017.

[4] J.W. Barrett, E. Süli, Existence and equilibration of global weak solutions to kinetic models for dilute polymers I: Finitely extensible nonlinear bead-spring chains, Math. Models Methods Appl. Sci., 21, 12111289, 2011.

[5] J.W. Barrett, E. Süli, Existence and equilibration of global weak solutions to kinetic models for dilute polymers II: Hookean-type bead-spring chains, Math. Models Methods Appl. Sci., 22, 1150024, 2012. 
[6] J.W. Barrett, E. Süli, Existence of global weak solutions to finitely extensible nonlinear bead-spring chain models for dilute polymers with variable density and viscosity, J. Differential Equations, 253, 3610-3677, 2012.

[7] J.W. Barrett, E. Süli, Existence of global weak solutions to compressible isentropic finitely extensible nonlinear bead-spring chain models for dilute polymers: The two-dimensional case, J. Differential Equations, 261, 592-626, 2012.

[8] J.W. Barrett, E. Süli, Existence of global weak solutions to compressible isentropic finitely extensible nonlinear bead-spring chain models for dilute polymers, Math. Models Methods Appl. Sci., 26, 469-568, 2016.

[9] R.B. Bird, C.F. Curtiss, R.C. Armstrong, O. Hassager, Dynamics of polymeric liquids, Fluid Mechanics, vol. 1, 2nd edn Wiley, New York, 1987.

[10] P.C. Bollada, T.N. Phillips, On the mathematical modelling of a compressible viscoelastic fluid, Arch. Ration. Mech. Anal., 205, 1-26, 2012.

[11] J.Y. Chemin, N. Masmoudi, About lifespan of regular solutions of equations related to viscoelastic fluids, SIAM J. Math. Anal., 33, 84-112, 2001.

[12] Q. Chen, C. Miao, Z. Zhang, Global well-posedness for compressible Navier-Stokes equations with highly oscillating initial velocity, Comm. Pure Appl. Math., 63, 1173-1224, 2010.

[13] Z. Chen, X. Zhai, Global large solutions and incompressible limit for the compressible Navier-Stokes equations, J. Math. Fluid Mech., 21, Art. 26, 23, 2019.

[14] P. Constantin, M. Kliegl, Note on global regularity for two-dimensional Oldroyd-B fluids with diffusive stress, Arch. Ration. Mech. Anal., 206, 725-740, 2012.

[15] R. Danchin, Global existence in critical spaces for compressible Navier-Stokes equations, Invent. Math., 141, 579-614, 2000.

[16] R. Danchin, A Lagrangian approach for the compressible Navier-Stokes Equations, Ann. Inst. Fourier, Grrenoble, 64, 753-791, 2014.

[17] R. Danchin, L. He, The incompressible limit in $L^{p}$ type critical spaces, Math. Ann., 366, 1365-1402, 2016.

[18] R. Danchin, J. Xu, Optimal time-decay estimates for the compressible Navier-Stokes equations in the critical $L^{p}$ framework, Arch. Ration. Mech. Anal., 224, 53-90, 2017.

[19] T.M. Elgindi, F. Rousset, Global regularity for some Oldroyd-B type models, Comm. Pure Appl. Math., 68, 2005-2021, 2015.

[20] C. Guillopé, Z. Salloum, R. Talhouk, Regular flows of weakly compressible viscoelastic fluids and the incompressible limit, Discrete Contin. Dyn. Syst. Ser. B, 14, 1001-1028, 2010.

[21] C. Guillopé, J.C. Saut, Existence results for the flow of viscoelastic fluids with a differential constitutive law, Nonlinear Anal., 15, 849-869, 1990.

[22] C. Guillopé, J.C. Saut, Global existence and one-dimensional nonlinear stability of shearing motions of viscoelastic fluids of Oldroyd type, RAIRO Modél. Math. Anal. Numér., 24, 369-401, 1990.

[23] Y. Guo, Y. J. Wang, Decay of dissipative equations and negative sobolev spaces, Comm. Part. Differ. Equ., 37, 2165-2208, 2012.

[24] B. Haspot, Existence of global strong solutions in critical spaces for barotropic viscous fluids, Arch. Ration. Mech. Anal., 202, 427-460, 2011.

[25] X. Hu, D. Wang, Global existence for the multi-dimensional compressible viscoelastic flows, J. Differential Equations, 250, 1200-1231, 2011.

[26] X. Hu, G. Wu, Global existence and optimal decay rates for three-dimensional compressible viscoelastic flows, SIAM J. Math. Anal., 45, 2815-2833, 2013.

[27] Z. Lei, Global existence of classical solutions for some Oldroyd-B model via the incompressible limit, Chinese Ann. Math. Ser. B, 27, 565-580, 2006.

[28] Z. Lei, N. Masmoudi, Y. Zhou, Remarks on the blowup criteria for Oldroyd models, J. Differential Equations, 248, 328-341, 2010.

[29] Z. Lei, Y. Zhou, Global existence of classical solutions for the two-dimensional Oldroyd model via the incompressible limit, SIAM J. Math. Anal., 37, 797-814, 2005.

[30] F. Lin, Some analytical issues for elastic complex fluids, Comm. Pure Appl. Math., 65, 893-919, 2012.

[31] P.L. Lions, N. Masmoudi, Global solutions for some Oldroyd models of non-Newton-ian flows, Chinese Ann. Math. Ser. B, 21, 131-146, 2000.

[32] C. Liu, N. Walkington, An Eulerian description of fluids containing visco-elastic particles, Arch. Ration. Mech. Anal., 159, 229-252, 2001. 
[33] Y. Lu, M. Pokorný, Global existence of large data weak solutions for a simplified compressible Oldroyd-B model without stress diffusion, Anal. Theory Appl., 36, 348-372, 2020.

[34] Y. Lu, Z. Zhang, Relative entropy, weak-strong uniqueness and conditional regularity for a compressible Oldroyd-B model, SIAM J. Math. Anal., 50, 557-590, 2018.

[35] A. Matsumura, T. Nishida, The initial value problem for the equations of motion of viscous and heatconductive gases, J. Math. Kyoto Univ., 20, 67-104, 1980.

[36] J. Oldroyd, Non-Newtonian effects in steady motion of some idealized elastico-viscous liquids, Proc. Roy. Soc. Edinburgh Sect. A, 245, 278-297, 1958.

[37] X. Pan, J. Xu, Global existence and optimal decay estimates of the compressible viscoelastic flows in $L^{p}$ critical spaces, Discrete Contin. Dyn. Syst. Ser. A, 39, 2021-2057, 2019.

[38] G. Ponce, Global existence of small solution to a class of nonlinear evolution equations, Nonlinear Anal. TMA., 9, 339-418, 1985.

[39] J. Qian, Z. Zhang, Global well-posedness for compressible viscoelastic fluids near equilibrium, Arch. Ration. Mech. Anal., 198, 835-868, 2010.

[40] M. Renardy, Local existence of solutions of the Dirichlet initial-boundary value problem for incompressible hypoelastic materials, SIAM J. Math. Anal., 21, 1369-1385, 1990.

[41] W.R. Schowalter, Mechanics of NonNewtonian Fluids, Pergamon Press, Oxford, 1978.

[42] R. Talhouk, Analyse Mathématique de Quelques Écoulements de Fluides Viscoélastiques, Thèse, Université Paris-Sud, 1994.

[43] W. Wang, H. Wen, On the Cauchy problem for an Oldroyd-B model in three dimensions, Math. Models Methods Appl. Sci., 30, 139-179, 2020.

[44] Z. Xin, J. Xu, Optimal decay for the compressible Navier-Stokes equations without additional smallness assumptions, arXiv:1812.11714v2.

[45] X. Zhai, The incompressible limit for the compressible Oldroyd-B model, Preprint.

[46] X. Zhai, Z. Chen, Global wellposedness to the $n$-dimensional compressible Oldroyd-B model without damping mechanism, arXiv:190\%.09829v1.

[47] X. Zhai, Z. Chen, Long-time behavior for three dimensional compressible viscous and heat-conductive gases, J. Math. Fluid Mech., 22, 38, 2020.

[48] Y. Zhu, Global classical solutions of 3D compressible viscoelastic system near equilibrium, arXiv:1809.04490.

[49] R. Zi, Global solution in critical spaces to the compressible Oldroyd-B model with non-small coupling parameter, Discrete Contin. Dyn. Syst. Ser. A, 37, 6437-6470, 2017.

$\dagger$ School of Mathematics and Statistics, Shenzhen University, Shenzhen, 518060, China

Email address: pingxiaozhai@163.com

$\ddagger$ School of Mathematics, South China University of Technology, Guangzhou, 510640, China

Email address: yshli@scut.edu.cn 